Abstract Silicon optical ring resonators are potentially valuable for many applications. Due to the limited design freedom (coupling coefficient and roundtrip length), the functionality and performance cannot always be fully explored and optimized. In addition, high-contrast silicon ring resonators suffer from parasitic coupling between the clockwise and counterclockwise modes as well as the parasitic coupling from the input to both circulating modes, which degrades or even distorts the response. In this paper, we give an overview of our work to harness these effects as additional design parameters to overcome the detrimental effects and realize novel functionalities in silicon ring resonators. Through simulations and experimental characterization, we show how the manipulation of backreflection and backcoupling enables various novel functions, including tunable Fano resonances with maximum slope rate over $700 \mathrm{~dB} / \mathrm{nm}$, tunable electromagnetically induced transparency which slows light down over 1100 ps, a single mode silicon ring resonator with a free spectral range over $150 \mathrm{~nm}$ and tuning efficiency over 11 times higher compared to that of a conventional silicon ring resonator, fundamental suppression of inevitable backscattering, spectral tuning, single sideband filtering, and ultra high $Q$ / large finesse resonances.

\title{
Using backscattering and backcoupling in silicon ring resonators as a new degree of design freedom
}

\author{
Ang $\mathrm{Li}^{1,2}$ and Wim Bogaerts ${ }^{1,2}$
}

\section{Introduction}

A ring resonator consists of a loop waveguide coupled to one or two bus waveguides. The nature of coherent feedback provided by the loop waveguide to the bus waveguide(s) generates resonances with high $Q$ factor and large extinction ratio (ER). The use of high-contrast silicon photonics as the platform for ring resonators further reduces the footprint as well as improves the free spectral range (FSR) and the finesse. Thus silicon ring resonators are considered for numerous photonics applications, ranging from laser cavities, filters, sensors, quantum optics, modulators, nonlinear optics and so on [1-5].

The basic schematic of an add-drop ring resonator is provided in Fig. 1, together with its transmission spectra. A very comprehensive review can be found in Ref. [6]. When designing resonators, there are usually only two parameters to manipulate, i.e. the coupling coefficient(s) $\kappa$ and the total roundtrip length $L$. For a given waveguide cross-section, $L$ determines the $F S R$ and resonant wavelength, while $\kappa$ plays a vital role in the $E R$ and $Q$ factor.

On one hand, this limited design space makes a ring resonator easy to model and design, but on the other hand,

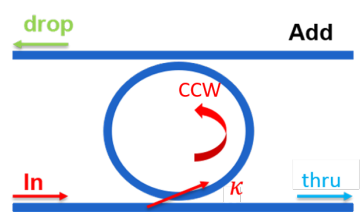

(a)

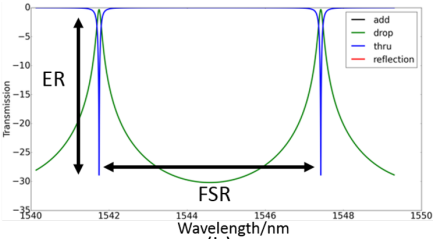

(b)

Figure 1: An ideal ring resonator generates Lorentzianshaped resonances at the through and drop ports.

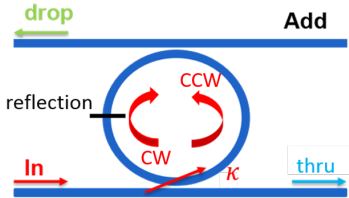

(a)

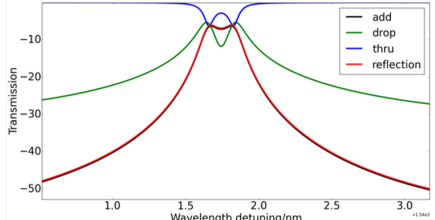

(b)
Figure 2: Internal reflections will impact the outputs of a ring resonator significantly. Resonances will split and strong transmission to the $a d d$ and in ports will appear.

1 Photonics Research Group, Ghent University-IMEC, Department of Information Technology, Ghent,9000 Belgium.

2 Center for Nano- and Biophotonics (NB-photonics), Ghent University, Belgium

* Corresponding author: e-mail: anl002@eng.ucsd.edu 


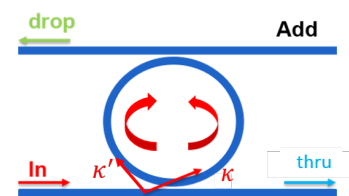

(a)

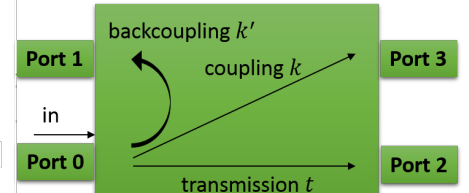

(b)

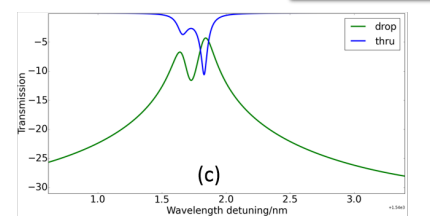

Figure 3: Backcoupling in a ring resonator a and a directional coupler (b). It is responsible for the asymmetry in the split resonance (c).

it limits the functionality and performance that can be achieved. Consequently, only symmetric Lorentzian resonances can be generated from a conventional ring resonator, and for a given type of waveguide there are fundamental upper limits on the achievable $Q$ factor, $E R$ and FSR [6].

To enhance the ring performance, variations on the classical ring resonator structure have been developed. For instance, silicon ring resonators with a Bragg grating reflector inside have shown to enhance the quality of the resonance [7]. Th Bragg reflector couples the clockwise and counterclockwise propagating modes in the ring, effectively turning the ring into a coupled resonator. This makes it possible to generate tunable fast and slow light [8], to generate a single mode in transmission [9] and reflection spectra [10], as novel optical sensors [11,12], and as filters with advanced functionalities [13,14]. Besides Bragg gratings, placing other reflective elements inside a ring cavity has also been frequently investigated, including tunable reflector, intentional defects, fiber tips, particles and so on. With these reflective elements, applications like complex resonances, exceptional point observation, chirality manipulation, single mode filtering can be achieved [15-18].

The use of multiple coupled ring resonators is very analogous to an internal reflector, as it also introduces coupling between different resonant modes. Coupled rings have been used to demonstrate filters with a tunable bandwidth, electromagnetically induced transparency (EIT), Fano resonance, tunable group delay, quantum information processing, novel sensors,optical single-sideband modulation and so on [19-23].

Even if there are no intentional reflective elements, there is still another source of internal reflections, namely the stochastic backscattering induced by sidewall roughness. This is an inevitable problem in high contrast waveguides, as used in most silicon photonics platforms [24-28]. The backscattering will cause weak and random reflections in the silicon waveguide, but in a ring resonator this effect has severe consequences, as all the weak reflections will coherently build up inside the resonator and form the countercirculating mode. The two circulating modes (clockwise and counterclockwise, indicated with $C W$ and $C C W$ ) are originally degenerate and resonate at the same frequency. When they start to couple with each other due to the backscattering,

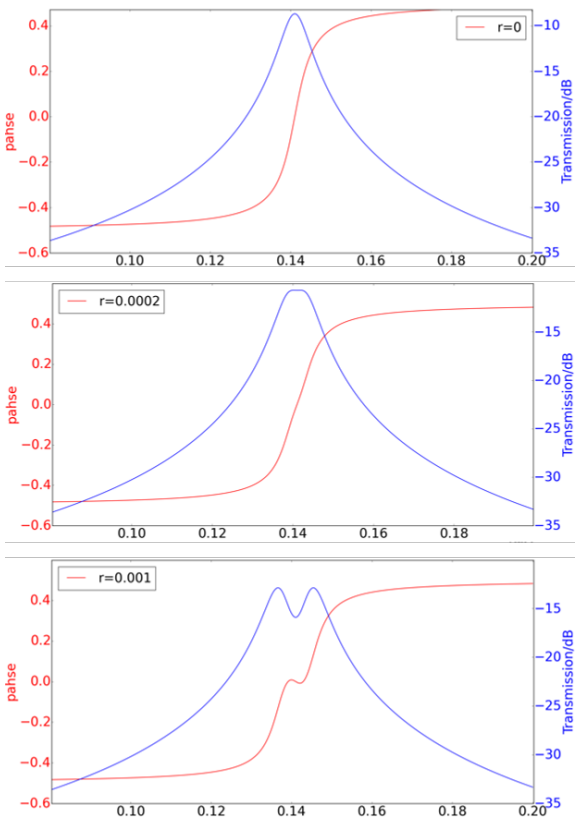

Figure 4: Internal reflections will impact the resonance of a ring resonator. $r$ in the figures refers to power reflectivity.

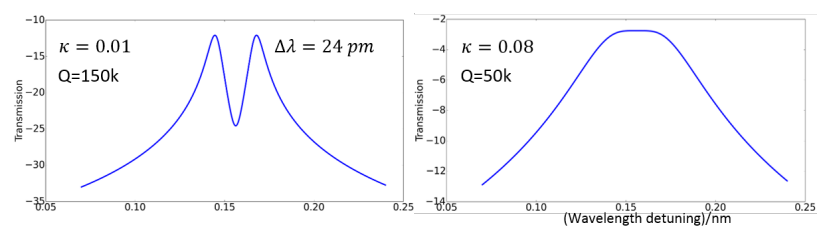

Figure 5: With increasing resonance bandwidth, the resonance splitting doesn't disappear, it just becomes invisible.

they will lose this degeneracy. As a consequence, resonance splitting will occur in the output spectra at the through and drop ports, and strong reflection to the in port and leakage to the $a d d$ port are also induced [29-31], which are shown in Fig. 2.

Irrespective of its origins, internal reflections impact the performance of a ring resonator. Therefore, there is a tremendous opportunity if the reflections can be engineered as a degree of freedom for the design of the ring resonator. It enables functions that cannot be realized with a ring resonator without reflections. Moreover, if the combination of internal reflections, including the stochastic backscattering, can be controlled, then the ring resonator can be tuned to a condition where the stochastic backscattering is suppressed. In later sections, we will show our methods to intentionally introduce reflections into a ring which can be fully tuned from from 0 to almost $100 \%$ using simple thermo-optic phase shifters; this is a key difference with previously mentioned approaches to intentionally introduce reflections inside a ring. We also demonstrated various functions using this technique, including backscattering suppression, tunable Fano resonances with ultra large extinction ratio and slope rate, 


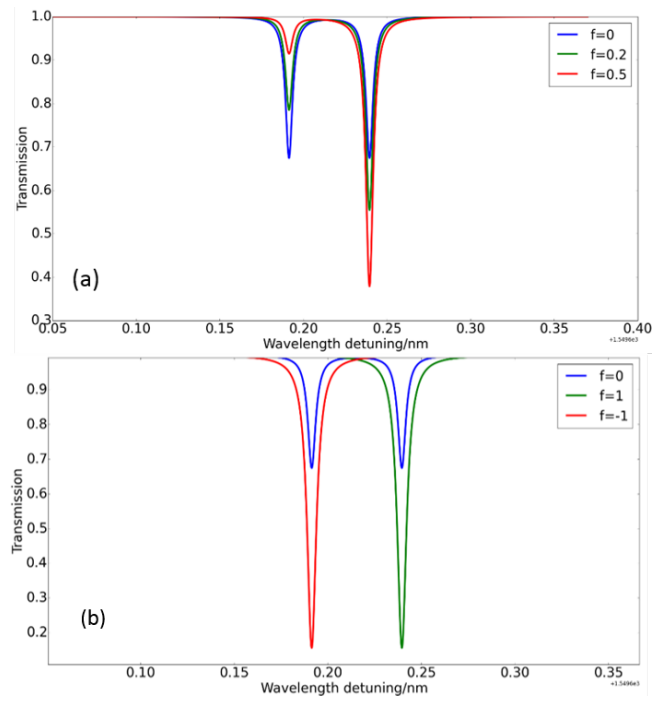

Figure 6: Simulated spectra at the through port with varying backcoupling ( $f$ factor) to show how the split resonance evolves.

tunable electromagnetically induced transparency, and slow light.

Even with the presence of internal reflections, there is another factor that also affects ring resonator behavior, namely the backcoupling. As illustrated in Fig. 3, backcoupling usually occurs unintentionally at the directional couplers of the ring [31]. Our research has demonstrated that this phenomenon is responsible for asymmetric resonance splitting. In other words, controlling the backcoupling allows tuning of the peak asymmetry in a split resonance, which is useful for resonance-splitting based applications. Later on, we will also demonstrate our approach to leverage this undesirable effect into another controllable degree of freedom for silicon ring resonators.

In this paper, we review and discuss our previous work presented in $[16,31-35]$ and present some additional results on these two novel degrees of freedom in the design and manipulation of ring resonators, which originate from two unwanted and non-deterministic parasitic effects (i.e. backreflection and backcoupling). By proper but non-trivial design, they can be turned into controllable parameters and thus can be engineered to achieve more advanced and complex functionalities from ring resonators. In section 2 , a theoretical explanation and experimental observation of backreflection and backcoupling will be given and their impact on the outputs of ring resonators will be be presented. After that, section 3.1 discusses ways to engineer one of the two parameters, namely internal reflections, and various advanced functionalities demonstrated in silicon ring resonators will be reported. Afterwards, section 3.2 will be about the manipulation of another degree of freedom, which is the backcoupling. Finally comes the conclusion.

\section{Unwanted and non-deterministic effects in silicon ring resonators}

An ideal ring resonator is supposed to support two degenerate modes, i.e. clockwise mode (CW) and counter-clockwise mode $(\mathrm{CCW})$. When exciting only a single input port, only one of these will be excited. This mode travels multiple roundtrips and lead to destructive (constructive) interference at the through (drop) port at resonance, which leads to the typical Lorentzian shaped peaks. However, unwanted internal reflections, usually through backscattering, as well as backcoupling (inadvertent excitation of the wrong mode in the coupling structure), can drmatically alter this behavior.

\subsection{Internal reflections}

Usually it's desirable for a ring to be implemented in a high index-contrast platform as this allows sharp bends, and therefore an ultra compact footprint, which in turn gives a large FSR and small mode volume. However, the high index contrast introduces the stochastic backscattering induced by the sidewall roughness. This problem has been extensively studied in previous decades as it is an inevitable consequence of the common fabrication process of lithography and etching. It has been reported to be the main loss source for silicon photonics strip waveguides and causes stochastic fluctuations to both reflection and transmission spectra of such a waveguide [24-27]. The effect of the backreflection gets amplified in resonators due to the long photon lifetime (and multiple roundtrips before decaying to a low intensity). Even very weak roundtrip reflection will coherently build up and excite the opposite circulating mode. Under such circumstance, the two originally degenerate circulating modes $(\mathrm{CW}$ and $\mathrm{CCW}$ ) will couple with each other and exchange power. Consequently their degeneracy will be broken and they will resonate on different frequencies as illustrated in Fig. 2 [29-31]. For a coupled resonator the same behavior (resonance splitting) is expected when the two resonators are identical. Very detailed and comprehensive models based on temporal coupled mode theory (tCMT) have been developed to explain this phenomenon $[29,31]$. Here we just give the transmission at the through port derived from tCMT:

$$
\begin{gathered}
\frac{S_{t}}{S_{i}}=1-\frac{1}{2}\left[\frac{\mu_{i}^{2}}{j\left(\omega-\omega_{1}\right)+\frac{B W_{0}}{2}}+\frac{\mu_{i}^{2}}{j\left(\omega-\omega_{2}\right)+\frac{B W_{0}}{2}}\right] \\
\omega_{1}=\omega_{0}+\mu_{r} \\
\omega_{2}=\omega_{0}-\mu_{r}
\end{gathered}
$$

Where $\omega_{0}$ and $B W_{0}$ are the intrinsic resonance frequency and bandwidth of the resonator without any parasitics. They are determined by the physical configurations including total length ,loss rate etc. $\mu_{i}$ refers to the mutual coupling between the resonator and the bus waveguide while $\mu_{r}$ represents the mutual coupling between the two modes (CW and CCW). Clearly, due to the existence of $\mu_{r}$, now two resonance 


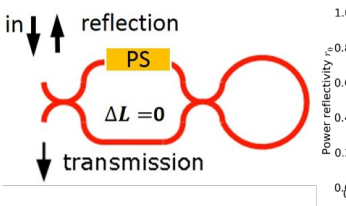

(a)

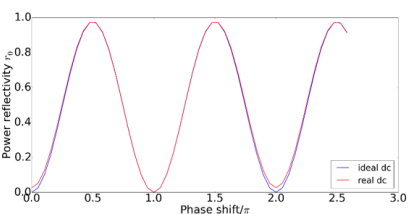

(b)
Figure 7: (a) schematic of an integrated tunable reflector. (b) Simulation results show that by adding only $0.5 \pi$ phase change to its arm, the reflectivity can be tuned from 0 to $100 \%$.

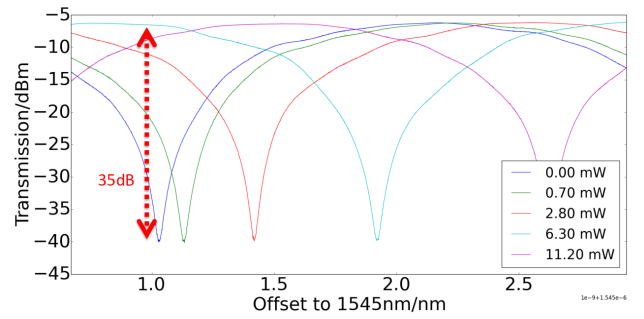

Figure 8: Experimental results of the tunable reflector shown in Fig. 7 confirm that by consuming only $6 \mathrm{~mW}$, the reflectivity can be tuned over a $35 \mathrm{~dB}$ change.

modes at individual frequencies appear and a resonance splitting appears.

On one hand, our previous research revealed that the resonance splitting caused by sidewall roughness induced backscattering can occur very frequently [31]. Especially for high $Q$ factor resonances, there can be as many as $80 \%$ resonances exhibiting splitting [31], which urges the need to take control of the backscattering. On the other hand, internal reflections can become beneficial if turn out to be controllable. This is very much similar with Zeeman or Stark effect in atom physics, where the spectral line of a energy level will split due to the presence of external magnetic or electric field that breaks the degeneracy of the energy states. Also it's very analogous to coupled harmonic oscillators in quantum physics, where the coupling strength and loss rate of each harmonic oscillator affects the frequency splitting condition of this coupled system [36]. Given the ease and convenience to measure a optical ring resonator, it can be useful to use such a ring resonator with two coupled modes or two coupled resonators to model those physics problems.

\subsection{Backcoupling}

Fig. 4, simulated with an optical circuit simulator - Caphe [38], clearly shows the impacts of internal reflections on the resonance of a ring resonator. First of all, it induces resonance splitting as already explained in paragraph above. Moreover, one might choose to reduce the $\mathrm{Q}$ factor (broaden the resonance linewidth) in order to avoid the resonance splitting. Actually this is incorrect as for a broader resonance, it's not the case that splitting disappears. Instead, the two split peaks become invisible under the envelope as evident in Fig. 5. For applications like filter, this might not be a problem depending on the signal bandwidth. However, for those applications where require precise control of the light-matter interaction like frequency comb generation, this is still problematic.

The maximum splitting distance is limited to one FSR, because then adjacent resonances will merge again. So theoretically one could control the reflectivity to tune the resonance over an entire FSR. Another noticeable fact from both Fig. 4 and tCMT model is the symmetry in the peak power of the split resonance. It's both shown in the simulated spectra and in tCMT model that, with only reflections, the splitting is always symmetric. This also explains why former research on modelling backscattering in silicon ring resonators can only describe symmetric splitting with conventional tCMT model [7,39-41]. However, our analysis of a very large number of measured ring resonances shows that the asymmetric splitting is dominant over symmetric splitting. In our dataset, we observed $80 \%$ of split resonances exhibiting asymmetric peak power [31]. The origin for the asymmetry is attributed to the backcoupling at the directional couplers [31], which describes how much light is coupled from input to the $\mathrm{CW}$ mode while the normal forward coupling represents the coupling strength from input to the CCW mode as shown in Fig. 3. Mathematically it can also be proven using a modified tCMT model. The following two equations show the transmission at the through port with backcoupling included:

$$
\begin{aligned}
& \frac{S_{t}}{S_{i}}=1-\frac{1}{2}\left[\frac{\left(\mu_{i}-\mu_{i}^{\prime}\right)^{2}}{j\left(\omega-\omega_{1}\right)+\frac{B W_{0}}{2}}+\frac{\left(\mu_{i}+\mu_{i}^{\prime}\right)^{2}}{j\left(\omega-\omega_{2}\right)+\frac{B W_{0}}{2}}\right] \\
& \frac{S_{t}}{S_{i}}=1-\frac{1}{2}\left[\frac{\mu_{i}^{2}(1-f)^{2}}{j\left(\omega-\omega_{1}\right)+\frac{B W_{1}}{2}}+\frac{\mu_{i}^{2}(1+f)^{2}}{j\left(\omega-\omega_{2}\right)+\frac{B W_{2}}{2}}\right]
\end{aligned}
$$

Where $\mu_{i}^{\prime}$ refers to the mutual coupling between the input and the $\mathrm{CW}$ mode through backcoupling, a very similar concept with the forward mutual coupling $\mu_{i}$. While $f$ in the equation (5) is a dimensionless factor representing the ratio between backcoupling and forward coupling. Using this factor gives a more straightforward view of how the two peak powers of a split resonance are affected by the backcoupling. In Fig. 6 we provide the simulation results of a ring resonator with the backcoupling included at the directional coupler. Clearly, varying backcoupling ( $f$ factor) can adjust the relative peak power of the split resonance. Interestingly, when $f=1$, in other words, the backcoupling equals the forward coupling, one peak will be suppressed, leading to a non-split resonance, which is also verified by equation (4). In the simulation, we manually modify the scatter matrix of the directional coupler in order to vary the backcoupling. However, this is completely unrealistic in reality. In section 3.2 we will describe a circuit for manipulating the backcoupling in silicon ring resonators. 


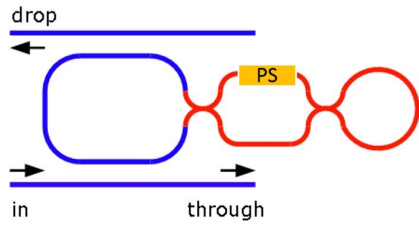

(a)

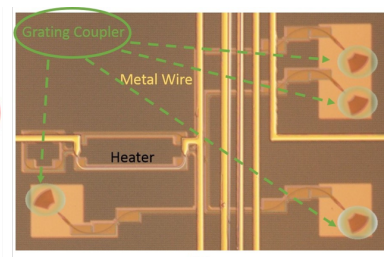

(b)
Figure 9: Schematic (a) and microscopic image (b) of a ring resonator with a tunable reflector inside.
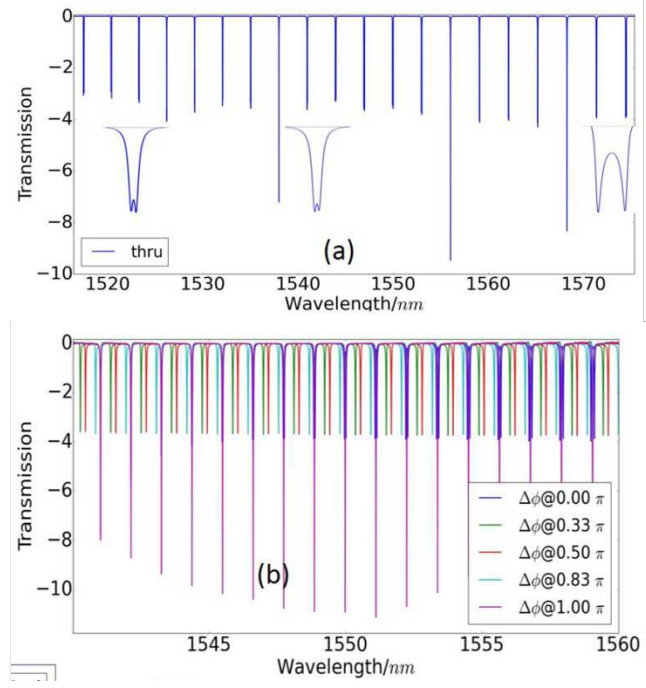

Figure 10: Simulated transmission at the through port of a ring resonator with various parasitics (a), and with both parasitics and a tunable reflector shown in Fig. 7 (b). By tuning the reflector to correct condition, the resonance splitting will be eliminated.

\section{Turn non-deterministic effects into controllable degrees of freedom}

Section 2 talks about the two detrimental and non-deterministic effects in ring resonators observed in rich experiments, namely internal reflections and backcoupling. Without proper correction, they are both detrimental to the performance of a ring resonator or any ring based devices. However, when they can be intentionally controlled, they could enable richer behavior in rings and be used to implement new advanced functionalities. In $[16,33,34]$ we initially proposed how to manipulate the internal reflections using tunable mirrors inside the ring, and with that demonstrated new behavior in ring resonators. For the backcoupling, we also proposed a technique in [35] to manipulate its effects. Below we describe these techniques and perform a further in-depth analysis on the merits.

\subsection{Engineering internal reflections}

Introducing internal reflections into a ring resonator is not new. These examples using Bragg gratings, defects, scatter-

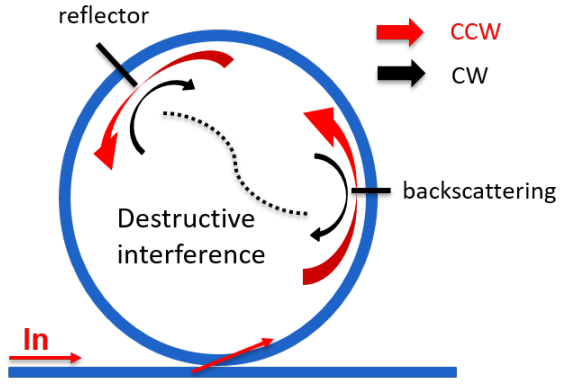

Figure 11: Principle to cancel the internal backscattering of a ring resonator using an intentional reflector.

ers or particles as reflective elements [7-15, 17, 18] listed in section 1 show that reflections in a ring can enable new functionalities, but they all exhibit some non-negligible drawbacks. Most importantly, the reflections introduced are not predictable and definitely not easily tunable. In other words, it's hard to consider them as a reliable degree of freedom at the design stage. Elaborate parameter sweeps are usually needed in order to obtain the expected results. Moreover, some of the demonstrated techniques are not compatible with CMOS process technology, which reduces the advantage in the context of silicon photonics. What's more, using metal particles or scatterers not only introduce reflections, but also often introduce extra loss. To properly engineer the reflections inside a ring, an integrated tunable reflector that has low loss, is simple to fabricate and has efficient tunability is needed.

\subsubsection{A tunable reflector}

In [32] we propose a 2-port integrated reflector as shown in Fig. 7. The reflector itself, which we proposed in [32], is a small circuit containing a Mach-Zehnder interferometer (MZI) with a loop waveguide connecting its outputs. The structure is simple, yet it can provide satisfying performance in terms of the tunability. The simulation in Fig. 7 shows that by adding only $0.5 \pi$ phase shift to one arm of the MZI, the reflectivity can be changed from 0 to almost $100 \%$. Also the experimental results of such a reflector confirms this broad and efficient tunability, which is plotted in Fig. $8.6 \mathrm{~mW}$ of electrical power is enough to change the reflectivity by as much as $35 \mathrm{~dB}$. So incorporating such a reflector inside a ring resonator can introduce controllable internal reflections.

\subsubsection{Ring with a single internal reflector}

The schematic and microscopic image of a ring resonator with such a tunable reflector is given in Fig. 9. In following section, we will show a couple of interesting applications using this device.

The first one comes about fundamental suppression of the stochastic internal reflections [33], including the inevitable sidewall roughness induced backscattering as well 

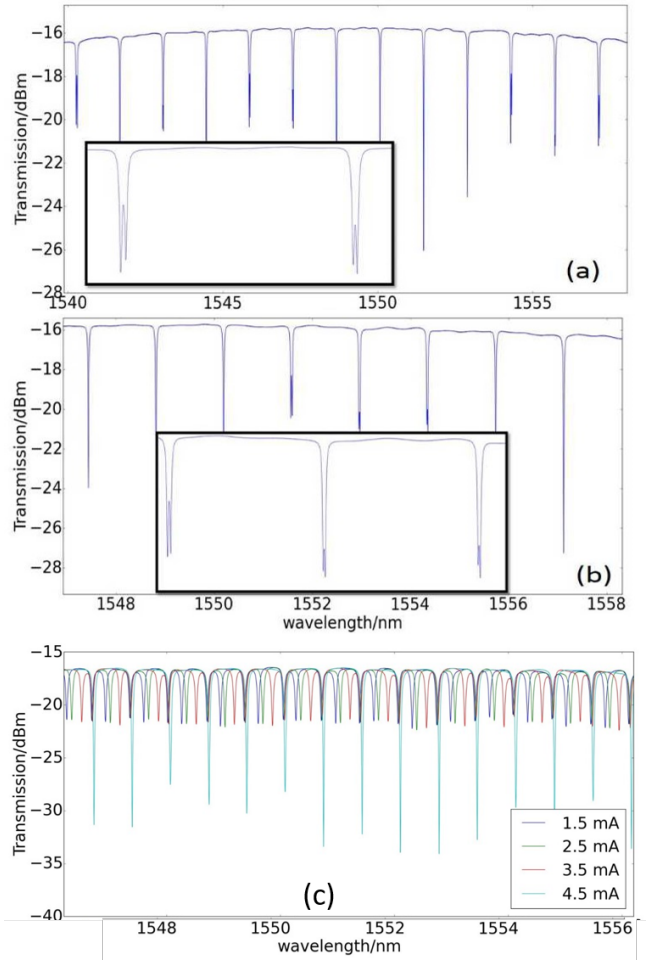

Figure 12: (a) and (b) present measured spectra at the through ports of two adjacent ring resonators without the tunable reflector to show presence of resonance splitting due to sidewall roughness. (c) provides the active measurement of a device with a reflector inside to show the elimination of the resonance splitting by tuning the reflector.

as lumped reflections induced at any perturbations or discontinuities, for instance the bend/straight interface and the coupling sections. The reflector is supposed to compete with the stochastic reflections. The principle behind this is simple as illustrated in Fig. 11: the stochastic backscattering will couple a portion of the $\mathrm{CCW}$ mode into the $\mathrm{CW}$ mode. And the intentional reflector will also couple another portion of $\mathrm{CCW}$ mode into $\mathrm{CW}$ mode. These two generated $\mathrm{CW}$ light can have destructive interference and cancel out each other under proper tuning condition of the reflector. Under this circumstance, the ring resonator behaves as if no stochastic backcoupling is present inside.

The simulation results using an optical circuit simulator Caphe [38] - are plotted in Fig. 10. In this circuit simulation, a lumped reflector is implemented in the ring cavity as a model for the stochastic reflections, which is responsible the resonance splitting. It has a constant reflectivity which is dependent on the ring length $[24,31]$ but a random phase that contains the stochastic characteristic. Other parasitics like the reflections and backcoupling at the couplers is also included. Fig. 10a shows the original state where resonance splitting is present due to the reflections. Clearly, by tuning the reflector into the correct condition, the splitting will be eliminated, as evident in Fig. $10 \mathrm{~b}$.
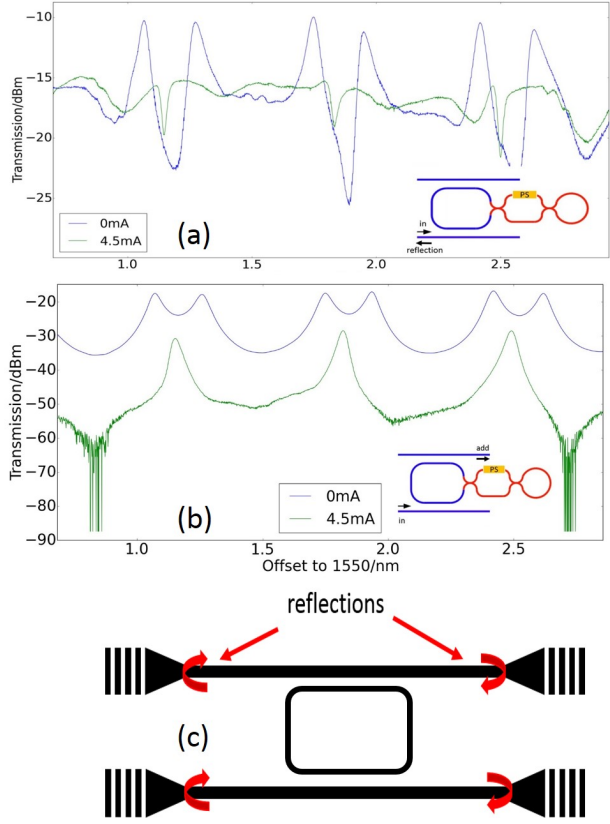

Figure 13: (a) and (b) give the measured reflection spectra and the unwanted leakage to the add port respectively. Under the correct tuning condition where splitting is eliminated, the reflection and leakage to the add port are significantly suppressed.

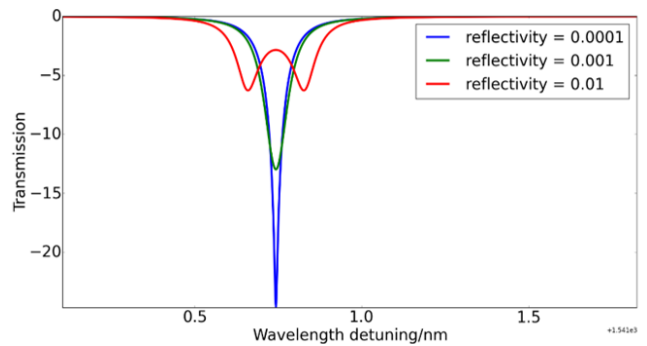

Figure 14: Strong internal reflections in a ring resonator will suppress the extinction ratio of a resonance.

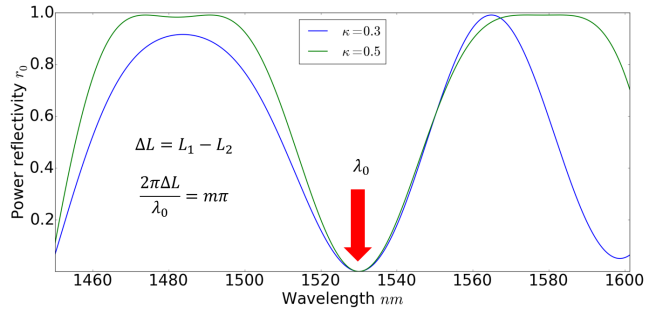

Figure 15: The reflection spectrum of the tunable reflector: only one wavelength has zero reflectivity while the rest in a broad span exhibits very strong reflections [32].

The experimental results are provided in Fig. 12 and Fig. 13. Obviously, not only the resonance splitting at the through and drop ports can be successfully eliminated, but 


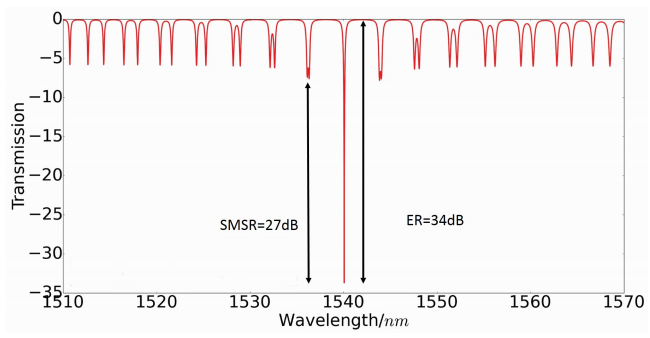

Figure 16: A silicon ring resonator with an ultra wide FSR by internal reflections engineering.

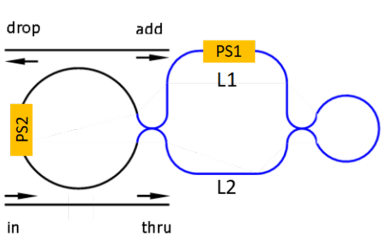

(a)

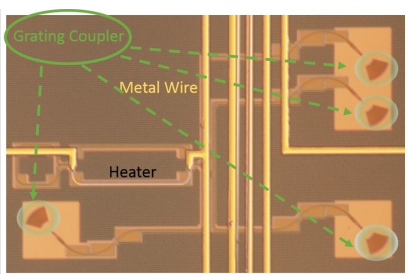

(b)
Figure 17: (a) and (b) give the schematic and microscopic image of the device used to generate a ring resonator with an ultra wide FSR and tuning range. $L_{1}, L_{2}$ refer to the armlength of the MZI reflector.

also the reflection to the in port and leakage to the add port can be significantly suppressed. The residual transmission at the add port is not indicating remaining internal backscattering. Instead, it is caused by the parasitic reflections outside the ring, for instance at the grating couplers. The Lorentzian shaped resonance of the residual transmission at the add port is actually another proof that there is indeed no backscattering inside the cavity, otherwise splitting should be present. Compared to other demonstrated approaches to solve the problem of backscattering [42], the tunable reflector has some significant benefits:

- It introduces much lower insertion loss to the circuit.

- It works for all ports of a ring resonator.

- It improves the extinction ratio of the resonance from its splitting condition.

However, the tunable reflector has a key drawback: because it is quite large, it limits the FSR of the ring due to the long roundtrip length. This can be alleviated somewhat by using more efficient (and therefore compact) phase tuners, but it remains a fundamental drawback of this approach. In section 3.2, we will introduce another method to avoid resonance splitting be manipulating the backcoupling, and which does not decrease the FSR of the ring.

Even though the reflector increases the roundtrip length, we can use the manipulation of the peak splitting to engineer the FSR of the ring. In fact, we demonstrated a ring resonator with an ultra wide FSR (over $100 \mathrm{~nm}$ ) and tuning range (over 11 times compared to a conventional silicon ring resonator) [37]. The principle behind this lies in the fact that strong internal reflections at a resonance will not lead to splitting but also degradation of its extinction ratio (ER) as illustrated

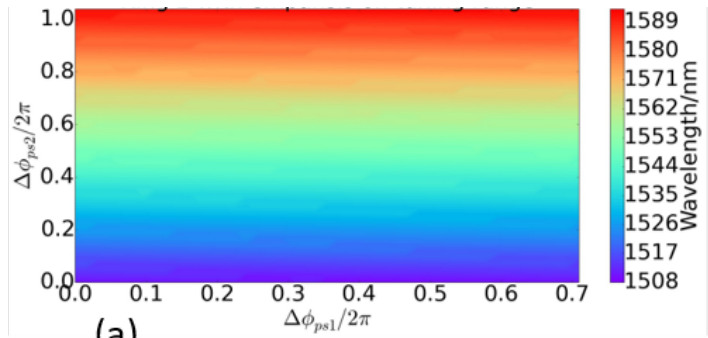

(a)

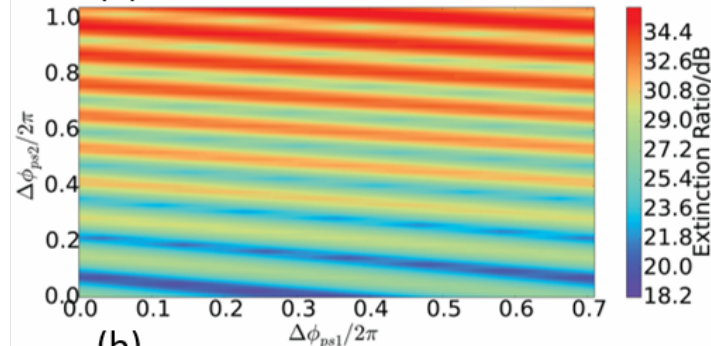

(b)

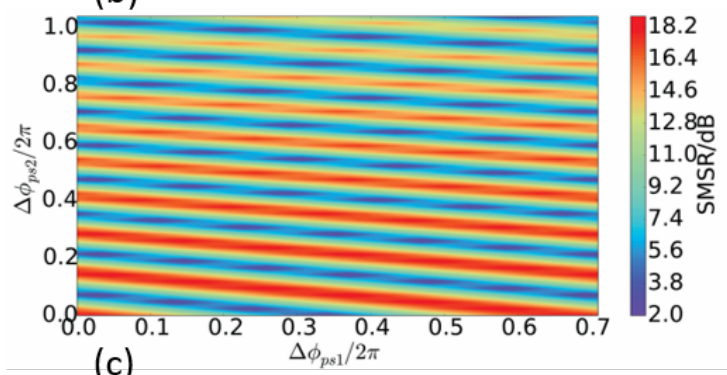

Figure 18: Contour plots of the tuning of the single mode in an ultra wide range. (a) presents the location of the single mode. (b) shows the extinction ratio and (c) plots the side mode suppression ratio in the tuning procedure. Figure reused from [32].

in Fig. 14 [32]. Also when $\Delta L=L 1-L 2=\frac{\lambda_{0}}{2}$ an unique reflection spectrum could be generated from our reflector, where only one wavelength point in a broad optical range has zero reflection (noted with $\lambda_{0}$ ), as shown in Fig. 15 [32]. When one resonance of the ring is aligned well at $\lambda_{0}$, only this resonance will exhibit a large ER and non-split shape, with all the rest showing splitting and small ER as plotted in Fig. 16. The schematic and microscopic image of such a device shown in Fig. 17 is similar with the previous one. They both consist of a ring resonator with a tunable reflector inside. The differences lie in two aspects: 1. For this device, the two arms of the reflector need to be different in order to construct a unique reflection spectrum. 2. There exist two phase shifters (PS1 and PS2 in Fig. 17) in order to provide an ultra wide tuning range. PS1 is responsible to shift the $\lambda_{0}$ of the reflector, at a much faster rate which is given by equation (6), while PS2 is in charge of shifting the entire ring spectrum to align one resonance to $\lambda_{0}$, which is governed by equation (7):

$$
\frac{\Delta \lambda_{r e f}}{\lambda_{0}}=\frac{\Delta n_{p s 1}}{n_{g}} \frac{L_{p s 1}}{\Delta L}
$$




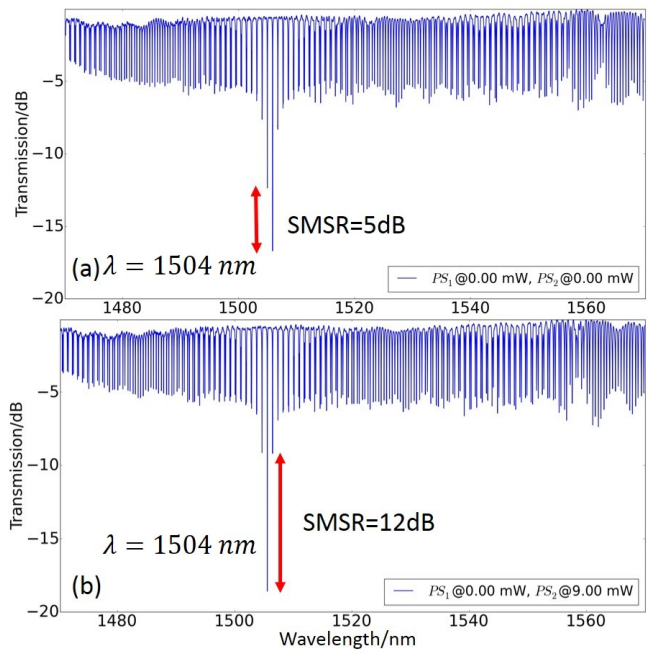

Figure 19: Measured spectra of the single mode ring based on the data reported in [37]. (a) shows the original condition where the ring resonance doesn't match the zero-reflection point perfectly. (b) gives the results when PS2 is tuned such that one resonance is well aligned to the zero-reflection point. The performance gets improved.

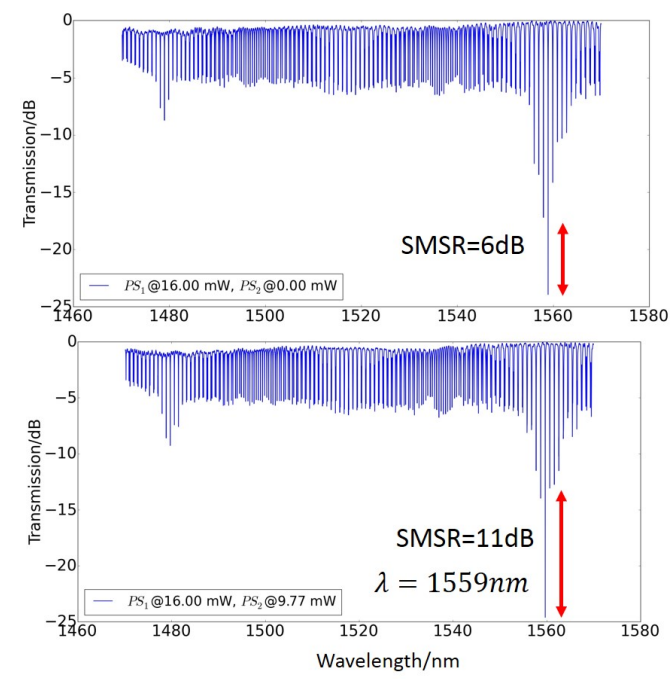

Figure 20: By controlling PS1, the zero-reflection point can be shifted. Then tuning PS2 will again make one resonance match it to get a good ER and SMSR. $16 \mathrm{~mW}$ (around 0.0148 index change) can shift the single mode around $55 \mathrm{~nm}$, which is $11 \times$ more efficient than a normal silicon ring resonator. Figure is based on the one reported in [37].

$$
\frac{\Delta \lambda_{\text {ring }}}{\lambda_{0}}=\frac{\Delta n_{p s 1} L_{p s 1}+\Delta n_{p s 2} L_{p s 2}}{n_{g}\left(L_{p s 1}+L_{p s 2}\right)+n_{e f f} L_{\text {rest }}}
$$

- $\Delta n_{p s 1}$ and $\Delta n_{p s 2}$ are the effective index changes in PS1 and PS2 respectively. $L_{p s 1}, L_{p s 2}$ refer to the physical length of these two phase shifters.
- $L_{\text {rest }}$ stands for the rest length of the ring resonator. $L_{\text {rest }}+$ $L_{p s 1}+L_{p s 2}$ represents the total length of ring $L$.

- $\Delta \lambda_{\text {ref }}$ and $\Delta \lambda_{\text {ring }}$ are the shift of $\lambda_{0}$ of the reflector and the resonance of the ring, respectively.

Clearly, the zero-reflection wavelength shifts much further with the same index change due to the amplification coefficient $\frac{L_{p s 1}}{\Delta L}$. For example, if $L_{p s 1}$ is $100 \mu \mathrm{m}$ while $\Delta L$ is set to be $6 \mu \mathrm{m}$, then $\lambda_{0}$ shifts over 16 times faster compared to a conventional resonance. This enables the possibility to achieve a wider tuning range of this single resonance. After $\lambda_{0}$ is shifted to a specific position, PS2 will be precisely tuned to arrive at a condition where $\Delta \lambda_{\text {ref }}=\Delta \lambda_{\text {ring }}+n \mathrm{FSR}$, then one resonance of the ring will match this new zero reflection point, thus a single mode at the new location is generated. Figure 18 provides the contour plots of the tuning of this single mode resonance by controlling PS1 and PS2. As shown in Fig. 18a, the wavelength can be tuned from $1508 \mathrm{~nm}$ til $1589 \mathrm{~nm}$ by less than $0.7 \pi$ phase shift added to PS1. Figure $18 \mathrm{~b}$ and Fig. $18 \mathrm{c}$ present the extinction ratio (ER) and side mode suppression ratio (SMSR) in the tuning period. They both can be maintained in a satisfying range $\left(\mathrm{ER}_{i} 20 \mathrm{~dB}, \mathrm{SMSR}_{\boldsymbol{i}} 13 \mathrm{~dB}\right)$.

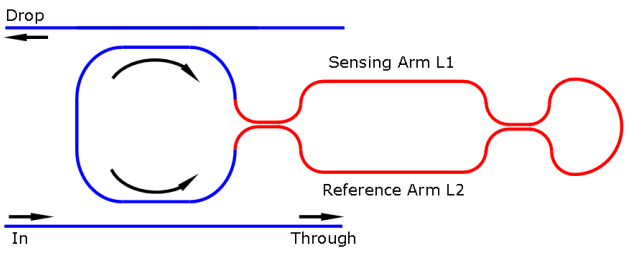

Figure 21: The schematic of the ring based sensor. One of the MZI reflector's arm will be exposed to the sensing environment.

The experimental characterization confirm the simulated performance, and both the ultra wide FSR and the tuning range can be achieved using two-step tuning mechanism via PS1 and PS2. Specifically, at the original state (Fig. 19a), we indeed observe an outstanding peak at $1504 \mathrm{~nm}$, however, as the ring resonance is not perfectly aligned to the zero-reflection point $\left(\lambda_{0}\right)$, the spectrum shows limited side mode suppression ratio (SMSR) and extinction ratio (ER). When we tune the PS2 to shift the entire ring spectrum a little bit, the resonance can reach an optimized condition with a large SMSR and ER as evident in Fig. 19b. Next stage is to tune the PS1 to shift $\lambda_{0}$ to a new location as plotted in Fig. 20a. By consuming only $16 \mathrm{~mW}$ (corresponding with an index change of 0.0148 or temperature change around $80 \mathrm{~K}$ ), the resonance can be shifted almost $55 \mathrm{~nm}$, from $1504 \mathrm{~nm}$ til $1559 \mathrm{~nm}$, which is 11 times more efficient than a conventional silicon ring resonator. Followed by a slight tuning of PS2, the SMSR and ER can be improved to the optimized condition as present in Fig. 20(b). We notice strong ripples in the off-resonance band. These ripples are those residual resonances of the resonator as the internal reflections could not fully suppress them to negligible conditions. They will be problematic for applications like WDM filters 
but still, there could be great candidate for sensors with ultra wide sensing range and single mode laser cavity with broad operation range.

Some other approaches using grating assisted coupler, bent coupler or metal particle deposition could also increase the FSR of a ring resonator $[14,15,43,44]$. However, grating usually requires high resolution in its spatial configuration, which is difficult to guarantee using current CMOS technology. While the metal particle deposition would significantly increase the cavity loss, thus decreasing the $Q$ factor. Also those approaches couldn't improve the tuning range of the resonance.

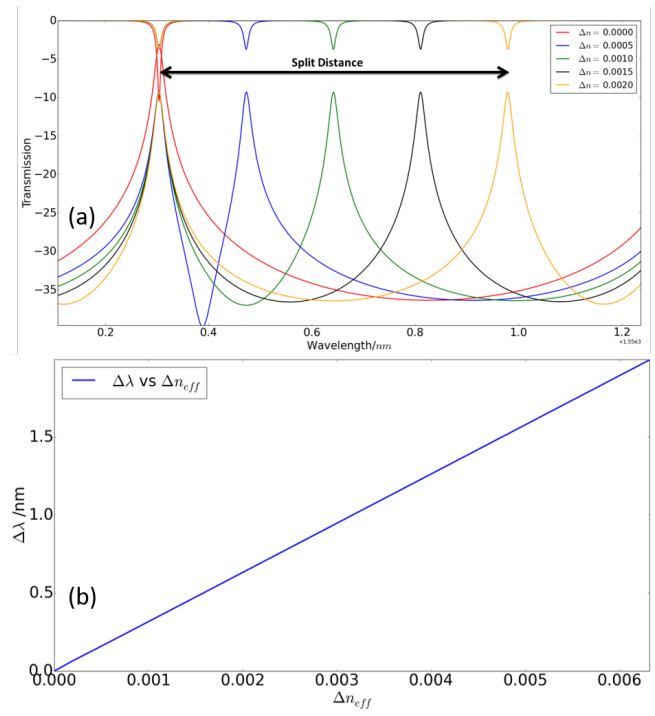

Figure 22: (a) Background index change will be converted to a change in the optical split distance. (b) Simulated $\Delta \lambda$ as a function of $\Delta n_{e f f}$. The efficiency is about $350 \mathrm{~nm} / \mathrm{RIU}$.

There are other possible applications for this device: for instance, it can be used as a very efficient sensor. Current silicon optical sensors are mainly based on an MZI structure or a ring resonator to convert the environmental index change to the optical spectrum change. MZI based sensors track the transmission change and usually have relatively low resolution due to the broad optical resonance, but they are temperature insensitive. It's the opposite case for ring resonator based sensors, who track the absolute resonance shift to provide high resolution, but they are very sensitive to ambient temperature variation. To combine their respective advantages and overcome their drawbacks, we propose to use a ring resonator with a reflector inside as a novel integrated optical sensor. One arm of the reflector serves as the sensing arm and is exposed to environment as shown in Fig. 21 with all the rest parts covered by protection layer. A reflector inside will cause resonance splitting at the output of the ring resonator. The principle is to convert the index change felt by the sensing arm to the change in the split distance. As the index change will lead to a change in the reflectivity, which will turn into a change in the splitting distance $(\Delta \lambda)$. In such way, this sensor is supposed to have both high resolution and temperature insensitivity, as the background temperature change will be cancelled out due to the nature of the balanced MZI. The simulated results are given in Fig. 22. It has an efficiency about 350nm/RIU (refractive index unit).

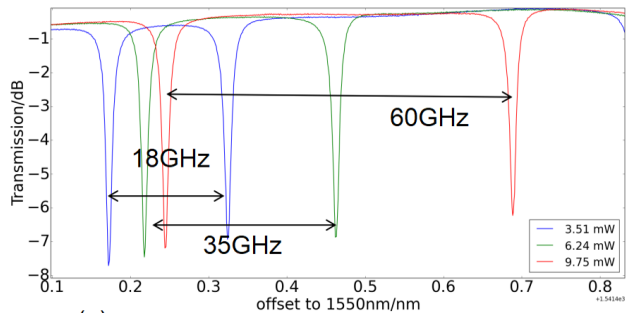

Figure 23: The distance between two peaks could be fully controllable by tuning the reflector, which could be potentially developed towards a tunable microwave filter.

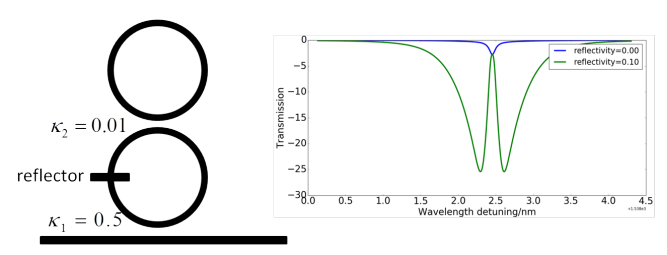

Figure 24: Implementing reflector in coupled-resonator structures could further boost the potential functionalities. Here is an example that coupled resonators with a reflector in one of them could be able to generate all-passive EIT with low insertion loss.

Besides being implemented as a sensor, such a device can also be potentially developed towards tunable microwave photonics filter. The two peaks of a split resonance can be used to filter the RF sidebands as their optical distance is fully tunable by controlling the reflector as plotted in Fig. 23. Moreover, it's well known that resonance splitting can be taken advantage for fast light applications, due to the anomalous dispersion brought by the splitting region [8]. As now we have the full control of the splitting condition of a resonance, we are supposed to fully tune the dispersion behavior, thus this device can also be engineered for various pulse advancement.

\subsubsection{Reflector in coupled resonators}

The examples above are all about the value of putting a reflector inside a single ring resonator. While, for many applications, ring resonators are implemented in the way that two or more resonators are coupled. Applications like slow light, electromagnetically induced transparency, high performance modulators, biosensors, PT-symmetry study, signal processing etc. [21, 22,45-47] Use of coupled resonators is very similar with putting reflector inside resonators as they are all about activating extra modes and enabling coupling between those modes. In contrast, using reflector could efficiently control the mode coupling. So implementing the 


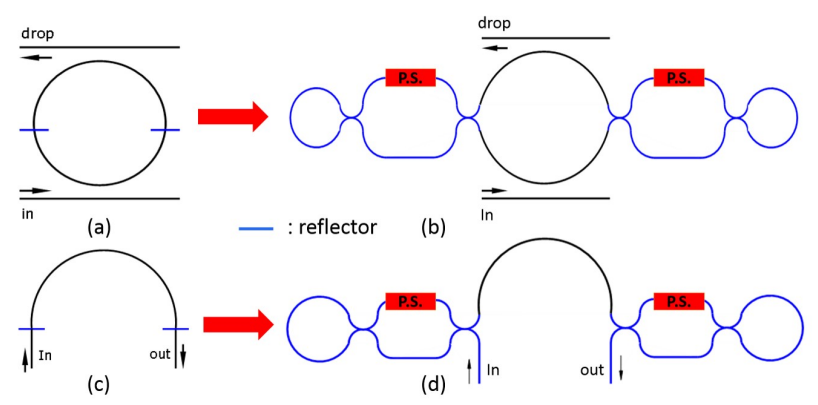

Figure 25: Conceptual schematic for a ring resonator with double reflectors inside as earlier proposed in [16]. Extra Fabry-Perot cavities will be formed inside the ring resonator.

tunable reflector inside those coupled-resonator structures could further boost the number of active modes and provide more diverse coupling between different modes. Given the large design space in coupled resonators with reflectors, we would not give a comprehensive characterization. Here, a simple example is provided to prove the value of introducing reflections into coupled-resonator strictures. It is a classical coupled resonators but with a reflector in the top resonator as shown in Fig. 24. Coupled resonators are popular choice to generate EIT and it usually requires one resonator to have an ultra high Q and the other to have very low Q $[48,49]$. This is very difficult to engineer properly in silicon photonics as losses are not trivial to manipulate. However, by putting a reflector in the top resonator, an all passive EIT could be generated as evident in Fig. 24.

\subsubsection{Ring with two internal reflectors}

Even though many things can be accomplished with a single tunable reflector inside the ring, it's natural to take this one step further: what if we put two reflectors inside a ring resonator as shown in Fig. 25. In such a case, which we presented in $[16,34]$, the device becomes more complicated as now it contains three cavities: the ring cavity and two FP cavities and they are coupled by the two reflectors. In brief, depending on the working conditions of the two reflectors, the device can work in four different regimes:

- A normal ring resonator. When both reflectors are tuned to introduce zero reflections, this device is just a normal ring resonator with Lorentzian-shaped resonances at its output spectra, as evident in Fig. 26a.

- Resonance splitting will appear when one of them start to introduce certain reflection as shown in Fig. 26a. In this regime, applications mentioned in former section can be achieved.

- Fano resonance: When the second reflectors also start to increase its reflectivity, two embedded FP cavities will be constructed. And their outputs or their modes are determined by two reflectors. Now the device becomes a mixed cavity system whose output is a result of the interaction of the FP cavity and the ring cavity. When the FP mode is tuned to low $Q$ factor and it's detuned to the

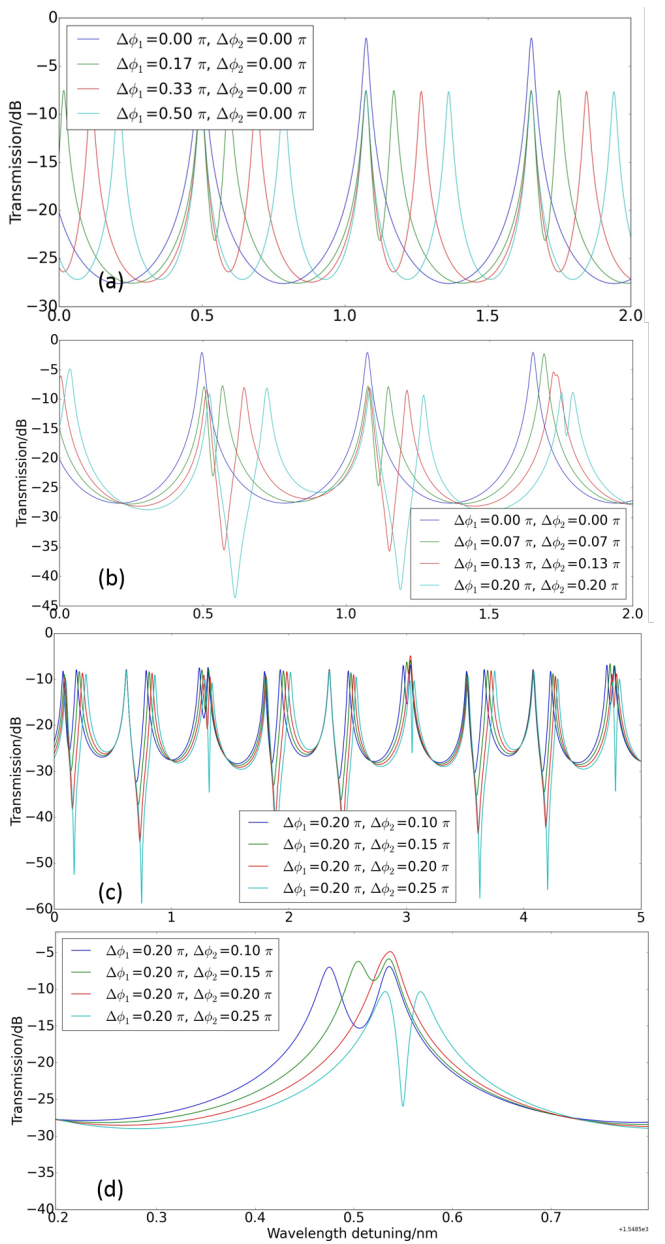

Figure 26: Simulated outputs at the drop port of the device with two reflectors inside. Multiple phenomenon can be generated by manipulating the two reflectors.

ring resonance, a Fano resonance will be generated as shown in Fig. 26c [16, 50]. The Fano resonance can be further developed towards ultra-high efficiency sensors, low power consumption switches, novel laser cavities etc.

- Electromagnetically induced transparency:On basis of the Fano resonance, if the two reflectors are further precisely tuned in order to get zero-detuning between the FP mode and the ring resonance, electromagnetically induced transparency (EIT) will be generated as plotted in Fig. 26d. It is the fundamental phenomenon for slow light applications.

Clearly, the working conditions of PS1 and PS2 strongly impact the generated resonance. To provide a more straightforward characterization of the device, we define two figures of merit of the resonance generated at the output: extinction ratio (ER) and slope rate (SR), as explained in Fig. 27. Also, two contour plots for these two figures of merit as functions of PS1 and PS2 are provided in Fig. 28. The $\mathrm{x}$ and $\mathrm{y}$ axis represent the phase shift added to PS1 and PS2, respectively. When PS1/PS2 increases from 0 to $0.5 \pi$, the reflectivity also increases from 0 to $100 \%$ accordingly. Besides the two 


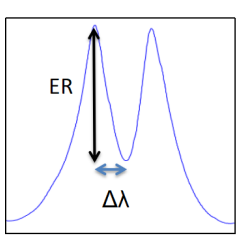

(a)

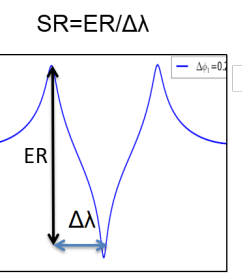

(b)

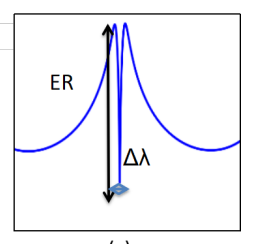

(c)
Figure 27: Two figures of merit to characterize the resonance generated from the device in Fig. 25. Middle panel shows the case of Fano resonance while right panel shows EIT pattern.

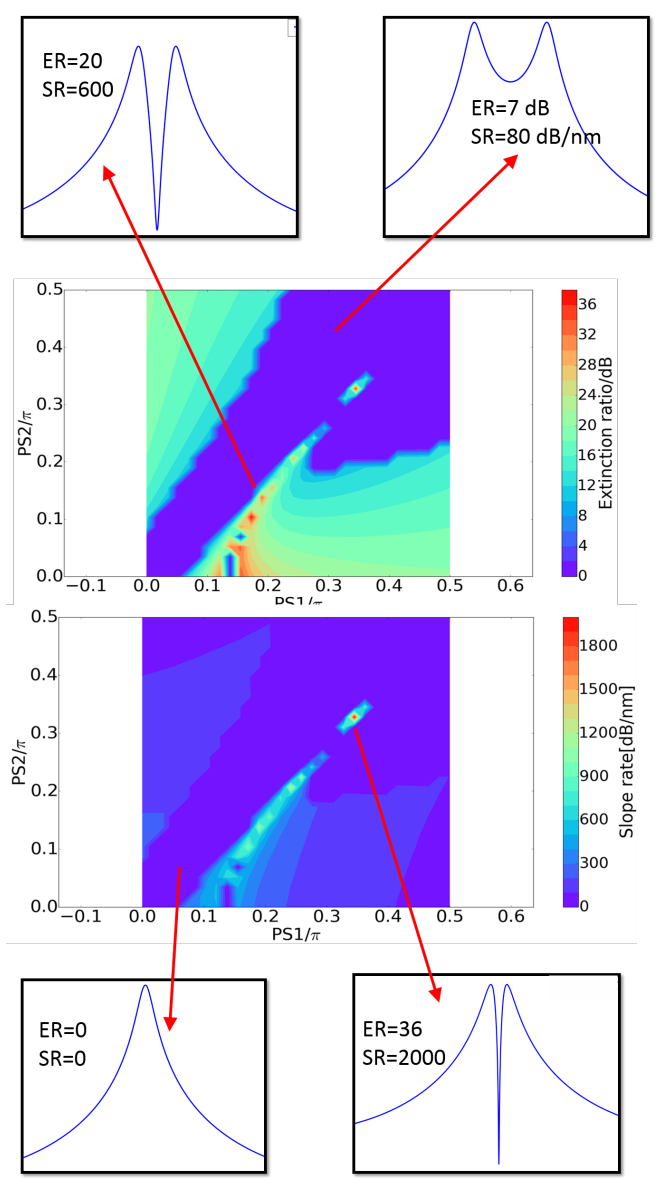

Figure 28: Contour plots of ER and SR vs PS1 and PS2. The $\mathrm{x}$ and $\mathrm{y}$ axis refer to the tuning range from 0 to $0.5 \pi$ of two phase shifters. They also represent the reflectivity of the reflectors from 0 to $100 \%$.

contour plots, four examples of from 4 different regions in the contour plot are also given. They refer to Lorentzian resonance, normal resonance splitting, Fano resonance and EIT, respectively. The former two belong to the large space of blue in the contour plots, where both ER and SR are low.

The microscopic figures of our devices are given in Fig. 29, and Fig. 30 presents the experimental results. Each spectrum in this figure corresponds with a tuning condition

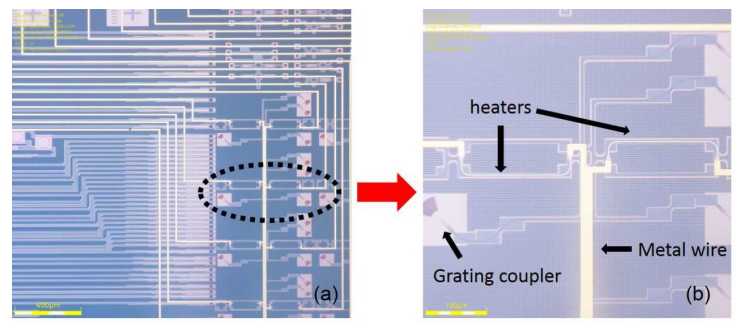

Figure 29: Microscopic images of our devices (a) and a zoomed view of the heaters (b).
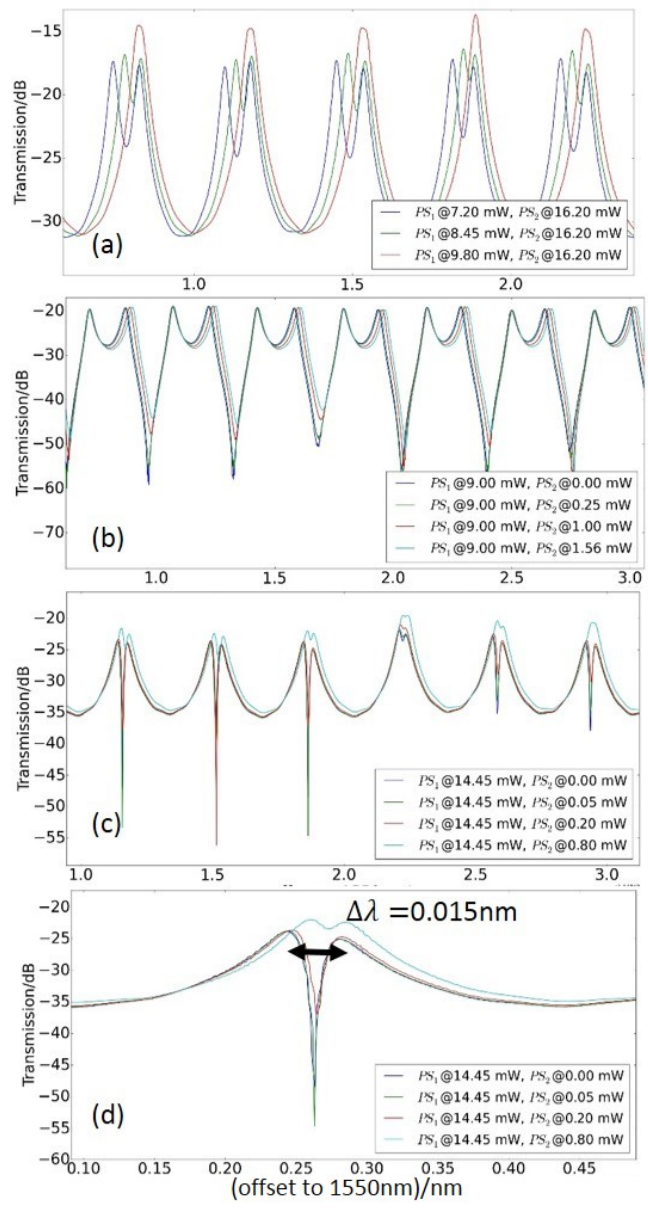

Figure 30: Overall measured spectra of our device with different tuning conditions of the two phase shifters. The figure presents a one-to-one correspondence with the three stages explained in the simulation part and plotted in Fig. 26.

of the two phase shifters. It satisfactorily reproduce the stages in the simulations, as shown in Fig. 26. In Fig. 30a, we plot the spectra of Lorentzian-shaped resonances where both reflectors introduce zero or very low reflections as well as the normal resonance splitting case where only one reflector introduces strong reflection. If we further increase the second reflector's reflectivity by changing the power injection to the phase shifter, the Fano resonance starts to 

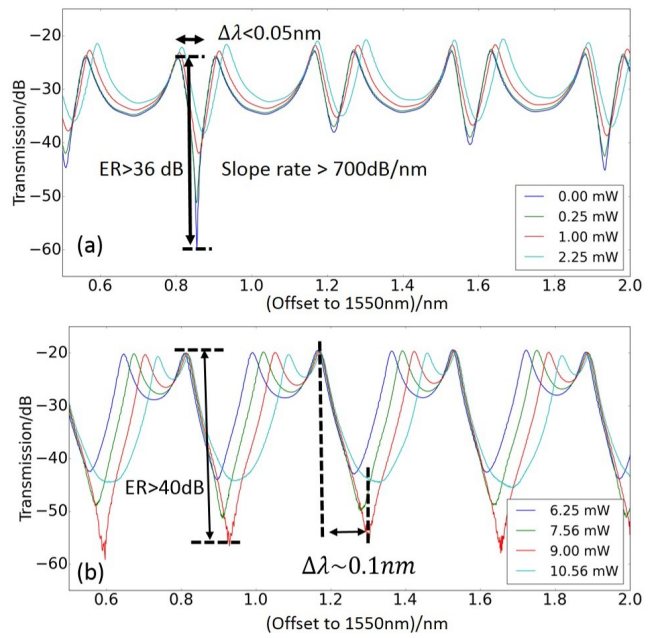

Figure 31: Measurements showing the tunability of the Fano resonance. One phase shifter is fixed with the other one is receiving different power injection. The maximum ER can be larger than $40 \mathrm{~dB}$ and the sharpest slope has a rate over $700 \mathrm{~dB} / \mathrm{nm}$.

appear, as shown in Fig. 30b. The spectra with EIT features is given in Fig. 30c and a zoom view of one EIT resonance is plotted in Fig. 30d. EIT is generated on the basis of Fano resonance, by precisely controlling one phase shifter. As we can see from the legend in Fig. 30c, the power injection to PS2 only changes from $0.0 \mathrm{~mW}$ to $0.8 \mathrm{~mW}$, but a clear EIT peak appears.

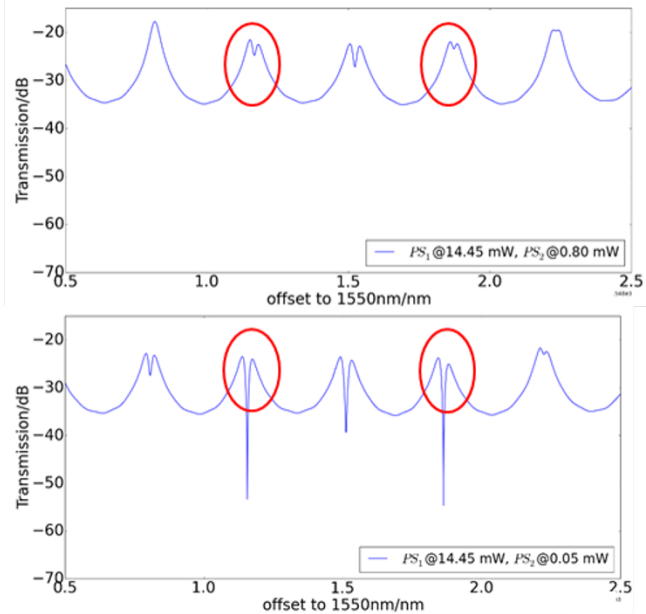

Figure 32: By precisely controlling one of the reflectors, a clear EIT peak emerges. Note the tiny change in the power injection shown in the legend.

Fig. 31 and Fig. 32 present specific characterizations of the generated Fano resonances and EIT, respectively. Both show efficient tunability through tuning the metal heaters. For the Fano resonances, a maximum extinction ratio over $40 \mathrm{~dB}$ and slope rate sharper than $700 \mathrm{~dB} / \mathrm{nm}$ are observed as evident in Fig. 31. Both values are, to our best knowledge, still the best achieved in Fano resonances demonstrated in
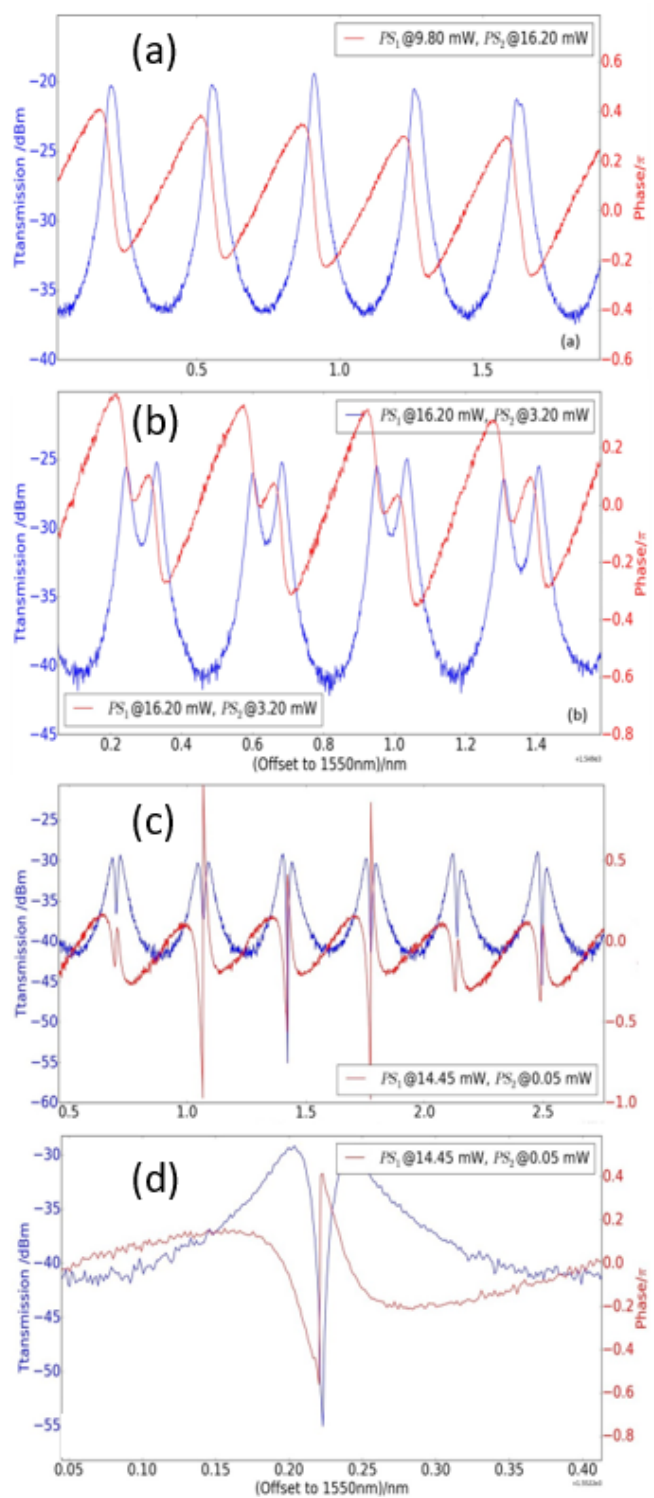

Figure 33: Measured phase response of this device as earlier reported in [34]. The abrupt phase change happens only at the EIT peak.

silicon photonics. The slope rate is over one order of magnitude higher than that of a normal Lorentzian resonance from a silicon ring resonator. Thus it enables high efficiency optical sensors and low-power consumption switches based on silicon ring resonators, as a much less wavelength shift is needed to achieve equivalent transmission change.

While for the EIT, it's desirable to implement it in silicon photonics as it is the fundamental phenomenon for slow light applications, like optical buffer or storage. Its key performance indicators lie in the group index around the EIT peak which is caused by the abrupt phase change within an ultra narrow optical span. So in contrast to the amplitude response, it is more interesting to study its phase response. To get the this, we use an optical vector network analyzer (OVNA) from Luna Inc. It has an integrated interferometer 


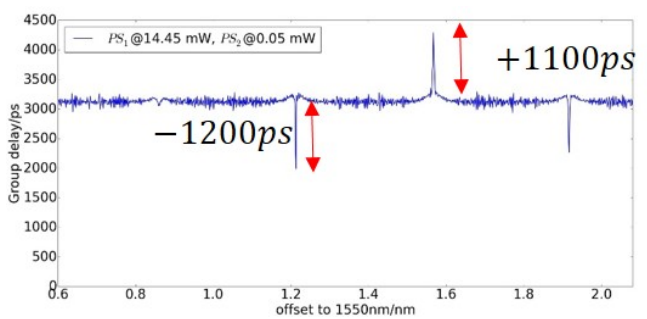

Figure 34: Measured group delay of the spectrum shown in Fig. 33c. At the EIT peak, there is a larger group delay at $4100 \mathrm{ps}$, compared to the background level at $3200 \mathrm{ps}$, the EIT slows light down at $1100 \mathrm{ps}$. We also notice some dips at the delay spectrum at the resonances showing splitting. This is the so-called fast light phenomenon. Due to our tunability of the internal reflections, we can achieve both tunable fast light and slow light.

that helps to extract the phase responses from our chip. The wavelength resolution is $1.5 \mathrm{pm}$. Fig. 33 plots the results, corresponding with the pure Lorentzian-shaped resonance pattern, normal resonance splitting pattern and EIT resonance pattern. Consistent with former literature, when it's pure ring resonance, the phase change only happens near the resonance wavelength [6]. While at the resonance splitting case, there is a gradual phase change in the splitting region. This phenomenon can be used to demonstrate fast light [8]. When EIT appears, there is an abrupt phase change as large as $0.95 \pi$ within an optical range around $10 \mathrm{pm}$. The extracted group index is as large as 350 , which is significantly larger than a conventional photonic crystal silicon waveguide.

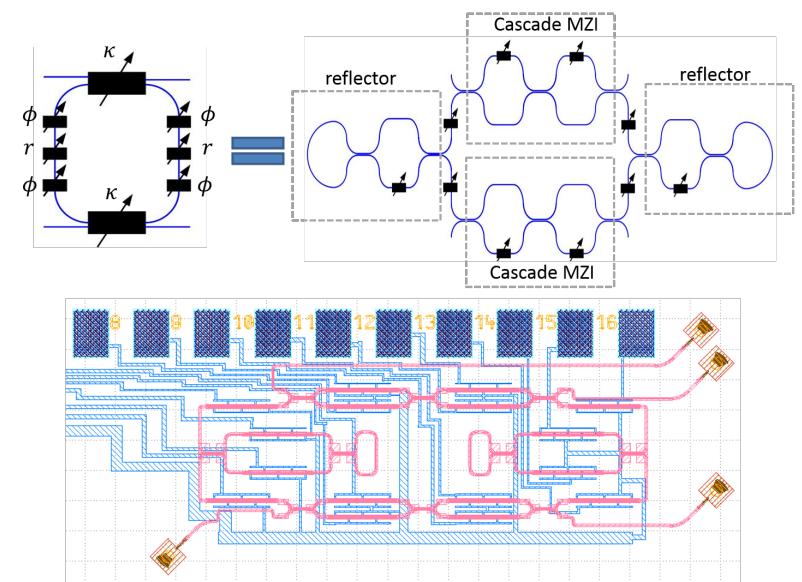

Figure 35: A generic programmable silicon ring resonator that has diverse degrees of freedom and is potential for rich functionalities. $\kappa$ represents the field coupling coefficient, $r$ refers to the field reflectivity of the reflector. $\phi$ stands for phase modulation.

We also give the measured group delay of such a device using the same equipment as plotted in Fig. 34. Clearly, at the EIT peak, the group delay is 1100 ps larger than the background level. We use a figure of merit named delay-

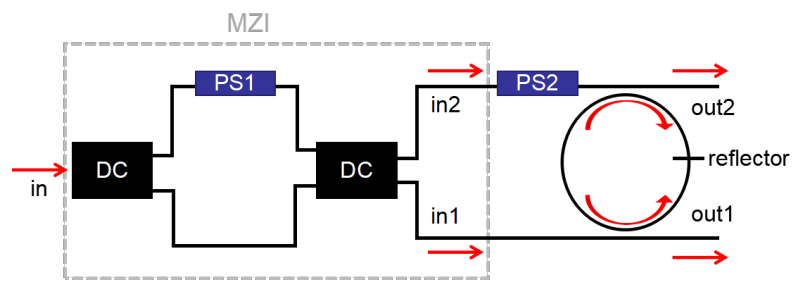

Figure 36: A circuit manipulate and counteract backcoupling.

\begin{tabular}{|c|c|c|c|} 
Device & Loss (dB/ns) & Delay (ps) & DBP \\
our device & 35 & 1100 & 9.67 \\
PhC cavity [51] & 85 & 17 & 0.16 \\
Cascade Resonators [19] & 65 & 30 & 0.94 \\
PhC Waveguide [20] & 35 & 85 & 8.5 \\
CROW [52] & 100 & 220 & 41 \\
Coupled Cavity [53] & 15 & 300 & 5.6
\end{tabular}

Table 1: A table compares different slow light structures.

bandwidth product (DBP) to compare with other slow light structures $[19,20,51-53]$. DBP is defined here as the product of delay in time $t$ and the bandwidth in frequency $\Delta \omega$. Our device demonstrates the largest delay and has a DBP of 9.67, which is only smaller than a CROW with 100 coupled resonators as shown in table 1 .

What's more, for the other peak where it shows resonance splitting, we observe a smaller group delay, indicating a pulse advancement. This is the so-called fast light effect introduced by resonance splitting [8]. Due our ability to fully control of the reflections inside the ring cavity (thus the resonance splitting conditions and the dispersion conditions within the splitting region), we are able to get a larger pulse advancement ( $1200 \mathrm{ps}$ in our case) than earlier results from literature [8].

\subsubsection{Generic programmable ring resonator}

With so many applications achieved from reflections engineering inside a ring resonator, the value of adopting it at the design stage has been proven. Thus we propose a so-called generic programmable ring resonator as shown in Fig. 35 . From the viewpoint of behavior model, it contains as many as 6 parameters that could be manipulated at the design stage, including the field coupling coefficients at the two couplers $\kappa$, field reflectivity $r$ from two reflectors, phase contrast between reflectors and the resonance wavelength of the ring resonator. While in terms of physical structure, each of those parameters could be tuned using a phase shifter. We choose to send it to IMEC to enjoy the mature CMOS fabrication technology and the phase shifter is designed as adjacent doped silicon waveguide with very high efficiency. For applications that require fast tuning, the phase shifter could be alternated to doped silicon waveguide with free carrier plasma effect. Note that, each coupler of the ring 
contains two cascaded MZI, in order to get universal tuning range of the coupling coefficient irrespective of the fabrication variation. The chip hasn't arrived yet but this ring is supposed to be able generate all the functionalities that reply on ring resonator with internal reflections, thus it's a much more powerful building block than a single ring resonator and it could be potentially be the basis for large scale programmable photonics integrated circuits.

\subsection{Engineering backcoupling}

As introduced in the section 2, besides the internal reflections, the backcoupling at the directional couplers also strongly impact the resonance of a ring resonator. When we want full control of a (split) ring resonance, we also need to be able to manipulate the backcoupling. As already discussed in section 2.2 with the help of Fig. 6 and equation (4), most rings experience backcoupling to a certain degree and the impact of backcoupling on the ring resonance cannot be neglected.

Backcoupling is caused by the directional coupler which couples a fraction of the optical power to the counterdirectional ring mode. There exist two contributions for the backcoupling at the couplers. First one is the two scatteres at the start and end of the couplers due to the perturbation caused by the adjacent waveguide. This contribution is relatively deterministic and is dominant when the couplers have short coupling length and small separation. While the other contribution, which is caused by the internal sidewall roughness is stochastic and becomes considerable when the coupling length is long because of the loose confinement in the coupling section and long interaction length of the field and sidewall roughness. While the directional coupler can be optimized to minimize the backcoupling, it is not straightforward to design a structure where we can actively control the backcoupling. Instead, we can obtain the same effect as backcoupling by controlling the ratio of how much the external light excites the $\mathrm{CW}$ and the $\mathrm{CCW}$ ring mode. In Fig.36 we picture a circuit, which we first presented in [35], that is designed to introduce and manipulate the backcoupling in a realistic way. It contains three parts. An MZI with an integrated phase shifter (PS1) is directly connected to the in port and splits it into two outputs: in 1 and in2. By tuning PS1, the ratio between the light intensity at ports in 1 and in 2 can be modified. The second part is another phase shifter (PS2), which is in charge of the phase difference between the light propagating from in 1 and in 2 . A third part is a ring resonator with certain (unknown) internal reflections that lead to resonance splitting, and with directional couplers that have a certain (unknown) amount of backcoupling. The form of the internal reflections can be either non-deterministic parasitic reflections (for instance, backscattering), but can also be controlled by a tunable reflector inside the ring.

At first sight, it's not straightforward to map this circuit to a ring resonator with backcoupling induced at the couplers, but the mechanism is similar. In a conventional ring resonator shown in Fig. 3 , the forward coupling $\kappa$ injects light into the counter-clockwise mode $(C C W)$, while the

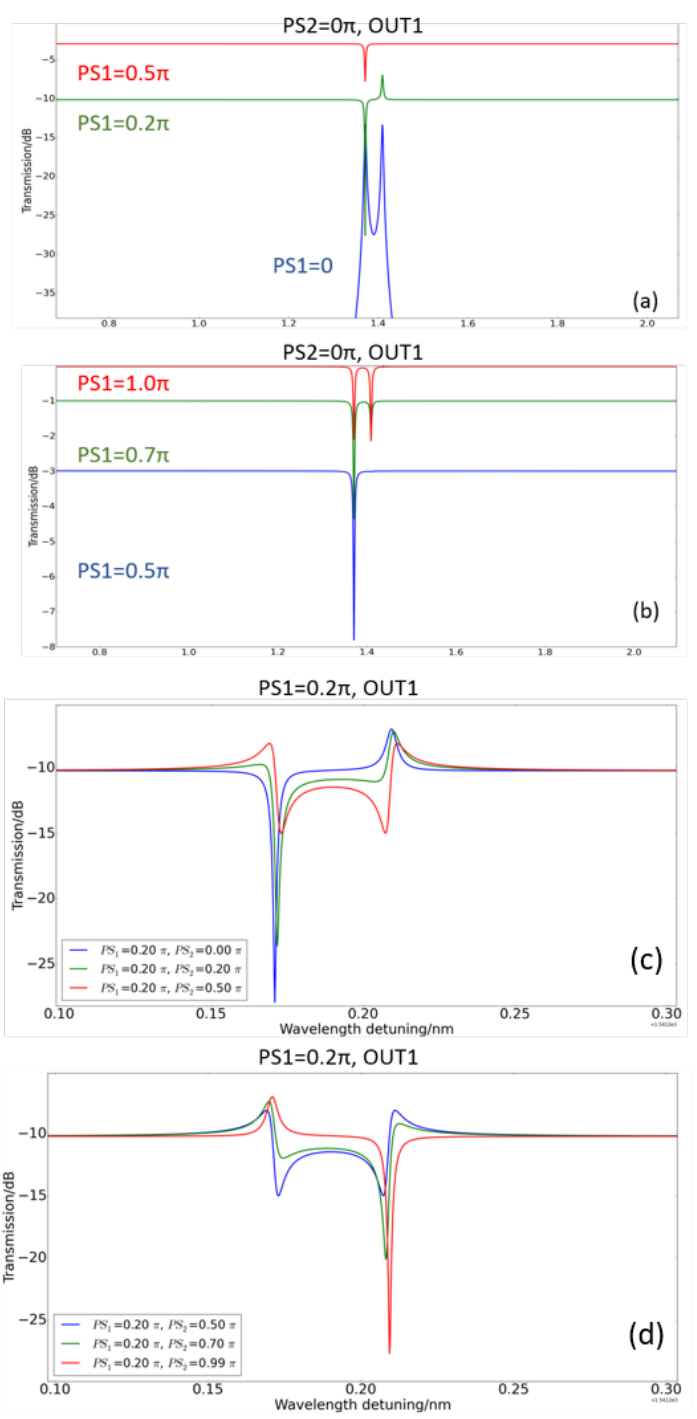

Figure 37: Simulated spectra show the impacts of PS1 and PS2. (a) and (b) show the results by varying PS1 and isolating PS2. (c) and (d) plot the resonances by varying PS2 and keeping PS $1=0.2 \pi$.

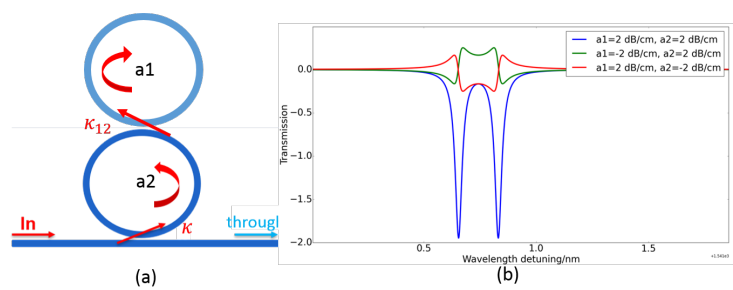

Figure 38: Schematic and simulated spectra of a coupledresonator circuit. Both resonators have identical eigenfrequencies but can have varying loss.

backcoupling $\kappa^{\prime}$ couples light from in port to the other mode $(C W)$. The amplitude and phase differences between $\kappa$ and $\kappa^{\prime}$ impact the resonance splitting. Similarly, in the circuit 
show in Fig. 36, the MZI splits input into two portions in 1 and in2. Each of them couples to one of the two circulating modes $(C W, C C W)$ through the directional couplers. In such a way, one of them serves as the forward coupling $\kappa$ while the rest one behaves as backcoupling $\kappa^{\prime}$. The split ratio of the MZI, which is controlled via tuning PS1, will then affect the relative amplitude difference between $i n 1$ and $i n 2$. Also tuning PS2 could effectively modify the phase difference between them. Thus, this circuit can fully control the power ratio and phase difference between the excitation of the $\mathrm{CW}$ and CCW mode. This way, the same effect as backcoupling can be achieved and the asymmetry in the split resonance can be controlled. Note that, even if the directional couplers in the ring have a certain amount of parasitic backcoupling, this can be fully compensated by the circuit.

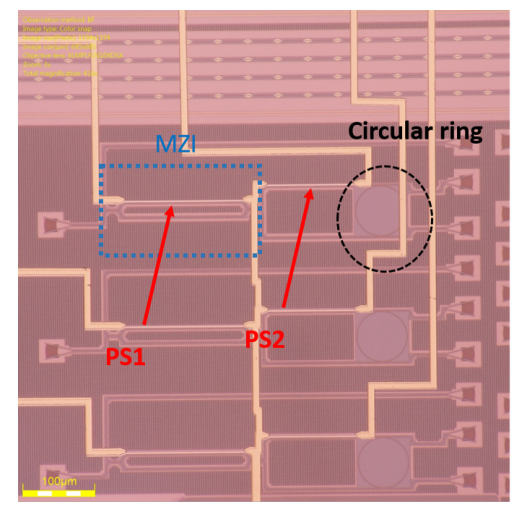

Figure 39: Microscope image of the devices to manipulate backcoupling.

To verify this, we build a corresponding optical circuit model in Caphe and run a series of simulations with varying key parameters, including the $Q$ factor of the resonator, the strength of internal reflections, the values of PS1 and PS2 etc. The directional couplers of the MZI are designed to be $0.5 / 0.5$, thus when $P S 1=0.5 \pi$, the MZI splits the light into two ports equally. In other words, the backcoupling has the same magnitude with forward coupling. Some examples of the output spectra at outl are plotted in Fig. 37. Those spectra at out 2 show similar trends thus are omitted here. Clearly, by varying PS1 and PS2, the split resonance change accordingly. Moreover, under the circumstances when $P S 1=1 \pm 0.5 \pi$ or $|\kappa|=\left|\kappa^{\prime}\right|$, one peak of the split resonance will be completely suppressed, resulting in a single resonance. This confirms the conclusion obtained in the introduction section, that is when the amplitude of backcoupling equals that of the forward coupling, one peak of the split resonance will be suppressed. All the simulations are consistent with the simulations of a pure ring resonator with backcoupling introduced by the couplers shown in Fig. 6 .

Another interesting effect is visible in Fig. 37c-d: when varying the phase shift in PS2, a pattern of double Fano resonances can be observed observed, each of which has an asymmetric shape and a sharp slope. This phenomenon is reported in a more complex coupled resonators system with asymmetric gain/loss as shown in Fig. 38 [54]. They

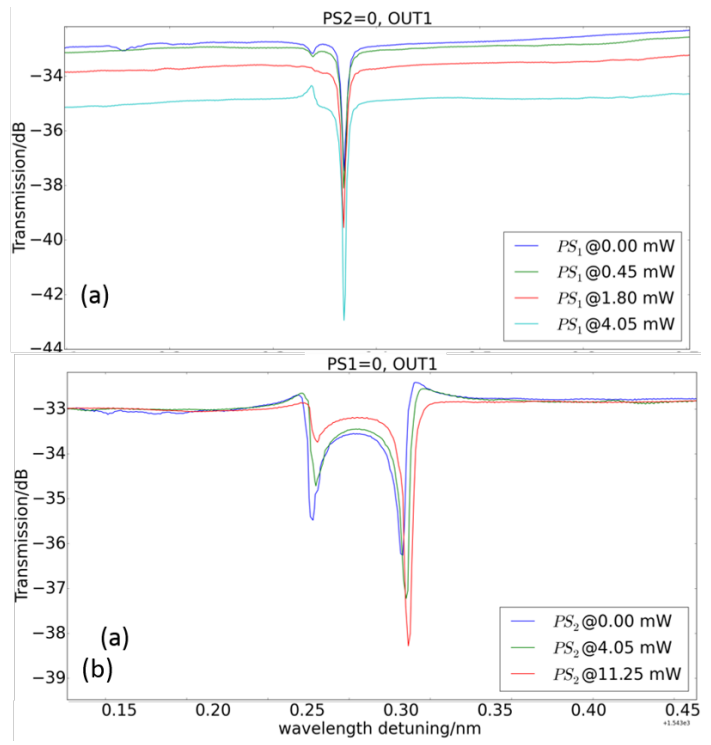

Figure 40: Experimental results of the device confirm the simulated features. By controlling the split ratio of the MZI, it's similar to modify the backcoupling of the ring resonator.

are designed to resonate at the same frequency. If both are lossy, only symmetric resonance splitting can be generated, while when one resonator has gain instead of loss, those sharp asymmetric Fano resonances will appear [54]. In other words, manipulating the backcoupling can be compared to manipulate the relative gain/loss of resonant mode. To make it more clear: the two modes ( $\mathrm{CW}$ and $\mathrm{CCW}$ ) in our circuit are equivalent to those modes ( $a 1$ and $a 2$ ) in the coupled resonator system. By default, the $\mathrm{CW}$ mode in our circuit is only excited by the CCW mode through their mutual coupling caused by backreflection in the ring. Similarly, the mode $a 2$ in the coupled resonator is also supported by mode a1 through internal reflections. The existence of coupling to $a 2$ provides extra contributions to $a 2$.

We confirmed these findings experimentally. We designed this circuit with a pure circular ring resonator with bend radius of $35 \mu \mathrm{m}$ in order to have sufficiently strong backscattering that can lead to resonance splitting, such that the effects of backcoupling would become visible. The fabricated device is shown in Fig. 39. Measured spectra with varying PS1 and PS2 are shown in Fig. 40. Also for simplicity, only spectra at out 1 are plotted. Clearly, we observe the resonance splitting, which is caused by the internal stochastic backscattering. The peak transmission of such a split resonance is indeed controlled by PS1 and PS2, or in other words, the phase and amplitude of the backcoupling, It confirms the backcoupling as a novel degree of freedom to modify the transmission of a ring resonator besides the index and coupling coefficient modulation. It could also be developed for potential applications like single-sideband filter, microwave photonics filter etc.

Also as shown in the red spectrum in Fig. 40a, one of the peaks disappears and resonance splitting is eliminated, indicating the backcoupling equals the forward coupling. This is consistent with the simulation results and could be used 


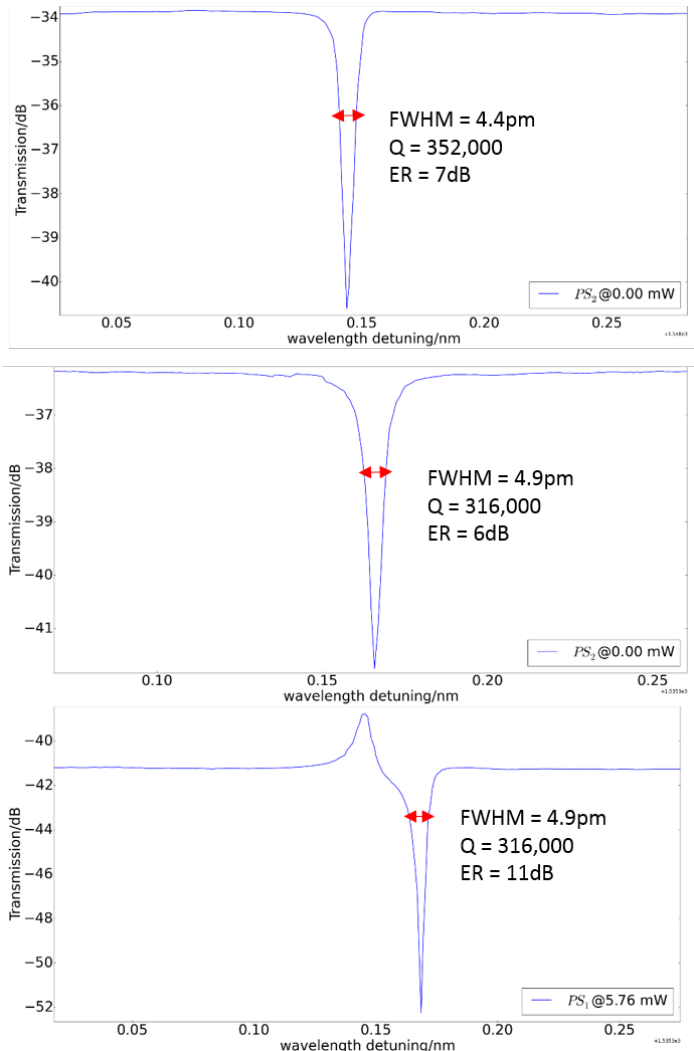

Figure 41: Details of measured resonances of a ring resonator with coupling gap at $400 \mathrm{~nm}$. All resonances show $Q$ factor larger than 300,000 and satisfying ER.The FSR of such a resonator is about $2.5 \mathrm{~nm}$. The calculated finesse is around 600 .

as an alternative method to solve the problem of backscattering induced resonance splitting. Compared to the method discussed in section 3.1.3, it wouldn't have a detrimental effect on the FSR of the ring resonator. Besides, tuning PS2 can also impact the resonance shapes and generate the double-wavelength Fano resonance pattern as evident in Fig. 40(b).

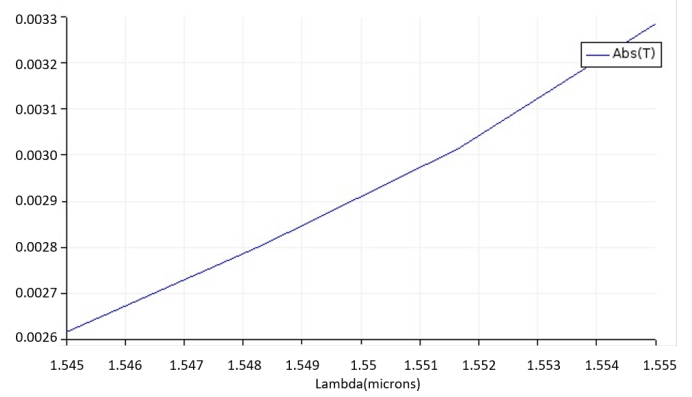

Figure 42: Details of measured resonances of a ring resonator with coupling gap at $400 \mathrm{~nm}$. All resonances show $Q$ factor larger than 300,000 and satisfying ER.The FSR of such a resonator is about $2.5 \mathrm{~nm}$. The calculated finesse is around 600 .
Another noticeable phenomenon is the ultra high $Q$ and large finesse of the resonances as evident in Fig. 41. All of them exhibit a bandwidth less than $5 \mathrm{pm}$, corresponding to $Q$ factors in excess of 300,000, sometimes reaching 400,000. Their extinction ratios (ER) are also between $6 \mathrm{~dB}$ and $11 \mathrm{~dB}$, which is a satisfying range. The FSR of such a resonator is around $2.5 \mathrm{~nm}$ and the corresponding finesse is around 600 . The FSR and finesse can be further increased by using smaller a bend radius. The value of $35 \mu \mathrm{m}$ we chose here is too large, as we want the stochastic backscattering to be sufficiently strong to cause resonance splitting, so we could observe the impact of backcoupling. For practical use, it can be safely reduced to $5 \mu \mathrm{m}$.

$$
Q_{i}=\frac{2 \pi n_{g}}{\alpha \lambda_{0}}=\frac{\lambda_{0}}{\alpha \times F S R \times R}
$$

We believe that the measured $Q$ factor of 380,000 is approaching the intrinsic $Q$ factor only limited by the cavity loss excluding the coupling loss to bus waveguides. As the mainstream cognition of the propagation loss of a silicon standard strip waveguide $(450 \mathrm{~nm} \times 220 \mathrm{~nm})$ fabricated with current CMOS technology (193 nm DUV lithography on $200 \mathrm{~mm}$ silicon wafer) is about $2 \mathrm{~dB} / \mathrm{cm}[55,56]$. A resonator with this propagation loss and a radius of $35 \mu \mathrm{m}$ correspond with an intrinsic $Q$ factor of 384,400 according to equation (8). Besides, the 3D FDTD simulation of the directional coupler in our ring resonator shows a power coupling coefficient less than 0.003, as evident in Fig. 42. Indeed, lowering the coupling coefficient is an effective method to improve the $\mathrm{Q}$ factor and ultimately, the intrinsic $Q$ factor could be reached. But for a conventional resonator with this low coupling coefficient, the resonances are almost invisible with an extremely small ER. The reason we got a large ER is not difficult to understand. For a conventional ring resonator shown in Fig. 1, it is the interference between the direct path at the through port and the roundtrip paths (CCW mode) coupled from the ring resonator to the bus waveguide that leads to a dip in the spectrum. When the two parties have similar strengths, the dip is more close to 0 and the resonance has a large ER. While for our device, at out 1 there are extra interference parties besides the two mentioned above. The light from in 2 couples to the $\mathrm{CW}$ mode, and this mode is coupled to the CCW mode due to internal reflections. If they are in phase with the CCW mode injected by inl, they will add up to destructively interfere with the light at the bus waveguide of out1. This is the reason why the resonance could maintain a large ER when approach the intrinsic $Q$ factor. Also we need to admit that, the higher $Q$ comes at the price of higher insertion loss of the device, as more light needs to be split into in 2 and coupled to the $\mathrm{CW}$ mode.

\section{Conclusion}

In this paper we gave an overview of our work in characterizing and manipulating the backreflections and backcoupling effects in silicon ring resonators, building on our work presented in [16,31-35]. We started with modelling and 
analyzing these detrimental and non-deterministic effects, which are confirmed through measurements on large numbers of ring resonators. We asses their impact through both simulations and experimental characterizations. Given their strong influence on the resonance of a ring resonator, we realize the importance of suppressing them, more importantly, the value of harnessing them as additional degrees of freedom for design of ring resonators.

Then we systematically proposed, discussed and demonstrated our work to manipulate the internal reflections and backcoupling in a realistic and efficient way. The internal reflections are engineered using integrated tunable reflector, whose reflection spectrum could be efficiently tuned using thermo-optic phase shifters. Various applications have been reported, including the fundamental suppression of inevitable backscattering caused by the sidewall roughness, tunable Fano resonance, tunable electromagnetically induced transparency and the slow light phenomenon, a single silicon ring resonator with an ultra wide FSR and tuning range, a novel optical sensor based on resonance splitting and so on. In terms of the backcoupling, we manipulate it using a dedicated circuit and confirm its impacts on a split resonance. Adding this as a design parameter for ring resonator could be of great benefit for various resonance splitting based applications. Moreover, it could be taken advantage to eliminate the backscattering induced resonance splitting and it is proven to push a resonance to its intrinsic $\mathrm{Q}$ factor without sacrificing its extinction ratio. From measurements, we observe resonances with $Q$ factor around 400,000 from a silicon ring resonator with strip waveguide cross-section and TE polarization, which to our best knowledge, is the largest value for this kind of ring resonator.

In summary, we believe that providing these additional degrees of freedom to ring resonator design could increase the range of functionalities that are enabled by rings, besides all the applications discussed here, and confirm the ring resonator as one of the most important components in integrated optics.

Key words: Resonators, silicon photonics, integrated optics, Fano resonance, slow light, high $Q$ cavity

Ang $\mathbf{L i}$ is a currently a postdoc scholar at the Ultrafast and Nanoscale Optics group at UCSD. Prior to this, he was a researcher in the Photonics Research Group at Ghent UniversityIMEC. in April of 2018, he obtained Ph.D from the same group. $\mathrm{He}$ has a strong interest in silicon photonics, particularly in resonant devices, fast and slow light, nonreciprocal transmission, high speed modulators and Fourier transform spectrometer.

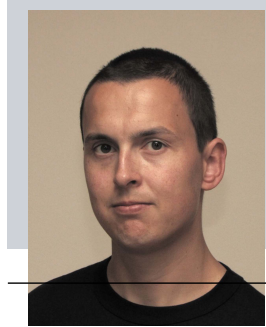

Wim Bogaerts is professor in the Photonics Research Group at Ghent University - imec. He received his $\mathrm{PhD}$ in the modelling, design and fabrication of silicon nanophotonic components at Ghent University in 2004. During this work, he started the first silicon photonics process on imec's $200 \mathrm{~mm}$ pilot line, which formed the basis of the multi-project-wafer service ePIXfab. Wim's current research focuses on the challenges for large-scale silicon photonics: Design methodologies and controllability of complex photonic circuits.

In June 2014, Wim co-founded Luceda Photonics, a spin-off company of Ghent University, IMEC and the University of Brussels (VUB). Luceda Photonics develops unique software solutions for silicon photonics design, using the IPKISS design framework.

Since 2016 Wim is again full-time professor at Ghent University, looking into novel topologies for large-scale photonic circuits, supported by a consolidator grant of the European Research Council (ERC).

Wim has a strong interest in telecommunications, information technology and applied sciences. He is a member of IEEE, the Optical Society (OSA) and SPIE.

\section{References}

[1] B. E. Little, S. T. Chu, H. A. Haus, J. Foresi, and J. P. Laine, Journal of lightwave technology 15(6), 998-1005 (1997).

[2] Q. Xu and M. Lipson, Optics letters 31(3), 341-343 (2006).

[3] F. Gardes, A. Brimont, P. Sanchis, G. Rasigade, D. MarrisMorini, L. O'Faolain, F. Dong, J. Fedeli, P. Dumon, L. Vivien et al., Optics express 17(24), 21986-21991 (2009).

[4] S. Azzini, D. Grassani, M. J. Strain, M. Sorel, L. Helt, J. Sipe, M. Liscidini, M. Galli, and D. Bajoni, Optics express 20(21), 23100-23107 (2012).

[5] M. Iqbal, M. A. Gleeson, B. Spaugh, F. Tybor, W. G. Gunn, M. Hochberg, T. Baehr-Jones, R. C. Bailey, and L. C. Gunn, IEEE Journal of Selected Topics in Quantum Electronics 16(3), 654-661 (2010).

[6] W. Bogaerts, P. De Heyn, T. Van Vaerenbergh, K. De Vos, S. Kumar Selvaraja, T. Claes, P. Dumon, P. Bienstman, D. Van Thourhout, and R. Baets, Laser \& Photonics Reviews 6(1), 47-73 (2012).

[7] Z. Zhang, M. Dainese, L. Wosinski, and M. Qiu, Optics express 16(7), 4621-4630 (2008).

[8] Q. Li, Z. Zhang, J. Wang, M. Qiu, and Y. Su, Optics express 17(2), 933-940 (2009).

[9] Q. Huang, K. Ma, and S. He, IEEE Photonics Technology Letters 27(13), 1402-1405 (2015).

[10] A. Arbabi, Y. M. Kang, C. Y. Lu, E. Chow, and L. L. Goddard, Applied Physics Letters 99(9), 091105 (2011).

[11] F. De Leonardis, C. E. Campanella, B. Troia, A. G. Perri, and V. Passaro, Sensors 14(9), 16017-16034 (2014).

[12] N. N. Klimov, S. Mittal, M. Berger, and Z. Ahmed, Optics letters 40(17), 3934-3936 (2015).

[13] A. Samarelli, A. Canciamilla, G. Morea, F. Morichetti, R. De La Rue, A. Melloni, and M. Sorel, Grating-assisted micro-ring resonators for silicon dual mode filters, in: The European Conference on Lasers and Electro-Optics, (2011), p. CK_P10.

[14] W. Shi, X. Wang, W. Zhang, H. Yun, C. Lin, L. Chrostowski, and N. A. Jaeger, Applied Physics Letters 100(12), 121118 (2012). 
[15] D. Urbonas, A. Balčytis, M. Gabalis, K. Vaškevičius, G. Naujokaitė, S. Juodkazis, and R. Petruškevičius, Optics letters 40(13), 2977-2980 (2015).

[16] A. Li and W. Bogaerts, APL Photonics 2(9), 096101 (2017).

[17] B. Peng, Ş. K. Özdemir, M. Liertzer, W. Chen, J. Kramer, H. Yllmaz, J. Wiersig, S. Rotter, and L. Yang, Proceedings of the National Academy of Sciences 113(25), 6845-6850 (2016).

[18] W. Chen, Ş. K. Özdemir, G. Zhao, J. Wiersig, and L. Yang, Nature 548(7666), 192 (2017).

[19] Q. Xu, S. Sandhu, M. L. Povinelli, J. Shakya, S. Fan, and M. Lipson, Physical review letters 96(12), 123901 (2006).

[20] A. Melloni, A. Canciamilla, C. Ferrari, F. Morichetti, L. O'Faolain, T. Krauss, R. De La Rue, A. Samarelli, and M. Sorel, IEEE Photonics Journal 2(2), 181-194 (2010).

[21] F. Morichetti, C. Ferrari, A. Canciamilla, and A. Melloni, Laser \& Photonics Reviews 6(1), 74-96 (2012).

[22] J. Wang, Z. Yao, T. Lei, and A. W. Poon, Scientific reports 4, 7528 (2014).

[23] S. Song, X. Yi, S. X. Chew, L. Li, L. Nguyen, and R. Zheng, Optical Engineering 55(3), 031114 (2015).

[24] F. Morichetti, A. Canciamilla, C. Ferrari, M. Torregiani, A. Melloni, and M. Martinelli, Physical Review Letters 104(January), 1-4 (2010).

[25] A. Li, Y. Xing, R. Van Laer, R. Baets, and W. Bogaerts, Extreme spectral transmission fluctuations in silicon nanowires induced by backscattering, in: Group IV Photonics (GFP), 2016 IEEE 13th International Conference on, (2016), pp. 160-161.

[26] Y. Xing, A. Li, R. Van Laer, R. Baets, and W. Bogaerts, Backscatter model for nanoscale silicon waveguides, in: 24th International workshop on Optical Wave \& Waveguide Theory and Numerical Modelling (OWTNM 2016), (2016).

[27] B. Peng, J. Rosenberg, W. D. Sacher, A. S. Jensen, M. Khater, W. M. Green, and T. Barwicz, Optics Express 25(19), 23477-23485 (2017).

[28] A. Li, T. Van Vaerenbergh, Y. Xing, P. Bienstman, W. Bogaerts et al., Experimentally demonstrate the origin for asymmetric resonance splitting and contributions from couplers to backscattering in soi microrings, in: Integrated Photonics Research, Silicon and Nanophotonics, (2015), pp. IM2B-6.

[29] B. E. Little, J. P. Laine, and S. T. Chu, Optics letters 22, 4-6 (1997).

[30] F. Morichetti, a. Canciamilla, M. Martinelli, a. Samarelli, R. M. De La Rue, M. Sorel, and a. Melloni, Applied Physics Letters 96(May), 13-15 (2010).

[31] A. Li, T. Vaerenbergh, P. Heyn, P. Bienstman, and W. Bogaerts, Laser \& Photonics Reviews 10(3), 420-431 (2016).

[32] A. Li, Q. Huang, and W. Bogaerts, Photonics Research 4(2), 84-92 (2016).

[33] A. Li and W. Bogaerts, Optics Express 25(3), 2092-2099 (2017).

[34] A. Li and W. Bogaerts, Optics express 25(25), 3168831695 (2017).

[35] A. Li and W. Bogaerts, Photonics Research 6(6), 620-629 (2018).

[36] L. Novotny, American Journal of Physics 78(11), 11991202 (2010).

[37] A. Li and W. Bogaerts, Optics letters 42(23), 4986-4989 (2017).
[38] M. Fiers, T. Van Vaerenbergh, K. Caluwaerts, D. V. Ginste, B. Schrauwen, J. Dambre, and P. Bienstman, JOSA B 29(5), 896-900 (2012).

[39] M. Moresco, M. Romagnoli, S. Boscolo, M. Midrio, M. Cherchi, E. S. Hosseini, D. Coolbaugh, M. R. Watts, and B. Dutt, Optics express 21(5), 5391-400 (2013).

[40] G. C. Ballesteros, J. Matres, J. Mart, and C. J. Oton, Optics Express 19(25), 24980-24985 (2011).

[41] S. Tallur and S. a. Bhave, Opt. Express 21(23), 2778027788 (2013).

[42] S. Werquin, S. Verstuyft, and P. Bienstman, Optics express 21(14), 16955-16963 (2013).

[43] R. Boeck, M. Caverley, L. Chrostowski, and N. A. Jaeger, An fsr-free silicon resonator reflector using a contradirectional coupler and a bragg reflector, in: Photonics North, 2015, (2015), pp. 1-1.

[44] N. Eid, R. Boeck, H. Jayatilleka, L. Chrostowski, W. Shi, and N. A. Jaeger, Optics express 24(25), 29009-29021 (2016).

[45] Y. Yang, S. Saurabh, J. Ward, and S. N. Chormaic, Optics letters 40(8), 1834-1837 (2015).

[46] B. Peng, Ş. K. Özdemir, F. Lei, F. Monifi, M. Gianfreda, G. L. Long, S. Fan, F. Nori, C. M. Bender, and L. Yang, Nature Physics 10(5), 394 (2014).

[47] N. L. Kazanskiy and P. G. Serafimovich, Optics Express 22(11), 14004-14013 (2014).

[48] D. D. Smith, H. Chang, K. A. Fuller, A. Rosenberger, and R. W. Boyd, Physical Review A 69(6), 063804 (2004).

[49] B. Peng, Ş. K. Özdemir, W. Chen, F. Nori, and L. Yang, Nature communications 5, 5082 (2014).

[50] A. Li and W. Bogaerts, A novel approach to create a tunable fano resonance with an extinction ratio over $40 \mathrm{db}$, in: Group IV Photonics (GFP), 2017 IEEE 14th International Conference on, (2017), pp. 27-28.

[51] S. Kocaman, X. Yang, J. McMillan, M. Yu, D. Kwong, and C. Wong, Applied Physics Letters 96(22), 221111 (2010).

[52] F. Xia, L. Sekaric, and Y. Vlasov, Nature photonics 1(1), 65 (2007).

[53] C. Hu, S. A. Schulz, A. A. Liles, and L. OFaolain, ACS Photonics 5(5), 1827-1832 (2018).

[54] Y. Lu, L. Xu, Y. Yu, P. Wang, and J. Yao, JOSA A 23(7), 1718-1721 (2006).

[55] P. Dumon, W. Bogaerts, V. Wiaux, J. Wouters, S. Beckx, J. Van Campenhout, D. Taillaert, B. Luyssaert, P. Bienstman, D. Van Thourhout et al., IEEE Photonics Technology Letters 16(5), 1328-1330 (2004).

[56] W. Bogaerts, R. Baets, P. Dumon, V. Wiaux, S. Beckx, D. Taillaert, B. Luyssaert, J. Van Campenhout, P. Bienstman, and D. Van Thourhout, Journal of Lightwave Technology 23(1), 401 (2005). 